\title{
ANALYSIS OF THE HEART-TORSO CONDUCTIVITY PARAMETERS RECOVERY INVERSE PROBLEM IN CARDIAC ELECTROPHYSIOLOGY ECG MODELLING
}

\author{
ABIR AMRI, MOURAD BELLASSOUED, MONCEF MAHJOUB, AND NEJIB ZEMZEMI
}

\begin{abstract}
In this paper, we prove a stability estimate of the conductivity parameters identification problem in cardiac electrophysiology. The propagation of the electrical wave in the heart is described by the monodomain model coupled to an elliptic equation describing the diffusion of the electrical wave in the whole body. Our result concerns both heart and torso conductivity parameters. The main difficulty that we solve in this paper is related to the transmission conditions between the heart and the torso. We first, establish Carleman estimates for the coupled heart-torso system. Then, using these estimates and the Bukhgeim and Klibanov approach, we prove a Lipschitz stability estimate of cardiac and torso conductivity parameters.
\end{abstract}

\section{INTRODUCTION AND MAIN RESULTS}

The electrocardiogram (ECG) is one of the most common tools in present-day medicine for the detection and diagnosis of a broad range of cardiac conditions. The ECG is a graphical representation of the electrical activity of the heart, which is enable to visualize the heart rhythm. Most of the common cardiac pathologies could be seen in the ECG traces. In particular slow conduction in the heart which is usually considered as a trigger of cardiac arrhythmia like atrial and/or ventricular flutters or fibrillation. These arrhythmia could lead to heart failure. The slow conduction in the heart is identified in the ECG when a QRS widening observed. Changes of the conductivities in the torso domain may also considerably affect the shape of the ECG. This is particularly important when the conductivities of the the organs surrounding the heart are severely modified. The cardiac tissue is a reactive conductive material. The reaction part is related to the electrical activity of the cardiac cells. The conductivity is an intrinsic behaviour of the biological tissue. Both reaction and conduction behaviour play an important role in the velocity of the electrical wave in the heart. Thus identifying, the cause of the changes in the conduction velocity if it is related to the reactive part or to the conductivities of the tissue and identifying its location in the heart domain may help in ameliorating the diagnosis of the heart condition. Consequently, an appropriate treatment could be delivered. In practice, today there are medical devices allowing to measure approximation of the electrical impedance in clinical interventions especially for cardiac radiofrequency ablation [31, 34]. The computed impedance in these devices does not provide the real conductivity of the tissue. Experimental works like [17, 32, 33] provide experimental estimation of the intracellular and extracellular conductivities. But the values of the conductivities may differ from an individual to another and thus these values have to be estimated for each patient. The mathematical modelling of the electrical phenomena in the heart provides a great opportunity to help solving these questions. In fact, the mathematical description of the propagation phenomena allows to provide a specific formulation of the conductive and reaction parts. This leads to a reaction-diffusion system known as the bidomain $[38,35]$ model or a simpler and most widely used monodomain model [16].

In this paper we are interested in studying the identifiability of the conductivity parameters. This study provides a theoretical analysis of the conductivity parameters identification stability in the heart and in the torso from measurements recorded in the a small region of the heart and from body surface measurements. As a consequence, we prove the uniqueness of these parameters . In the literature, Yan and Veneziani [42]

Date: November 23, 2020.

2010 Mathematics Subject Classification. Primary 35Q92, Secondary: 35R30.

Key words and phrases. Conductivity parameters, Cardiac electrophysiology, Carleman estimate, inverse problem, Stability estimate. 
use a variational procedure for the estimation of cardiac conductivities from measures of the transmembrane and extracellular potentials available at some sites of the tissue. Beretta et al. [11] developed a numerical approach to solve the inverse problem of detecting a spherical inhomogeneity from boundary measurements of the electric potential.

In this section, we will introduce our mathematical model for the electrical activity of the heart is the so-called monodoamin model. We assume that the intra- and extracellular conductivities $\sigma_{\mathbf{i}}$ and $\sigma_{\mathbf{e}}$ are proportional. Let the bulk conductivity tensor of the medium and the transmembrane conductivity tensor defined respectively as follows

$$
\sigma_{\mathbf{h}}=\sigma_{\mathbf{i}}+\sigma_{\mathbf{e}}, \quad \text { and } \quad \sigma_{\mathbf{m}}=\sigma_{\mathbf{i}} \sigma_{\mathbf{h}}^{-1} \sigma_{\mathbf{e}} .
$$

We assume that the cardiac domain $\Omega_{\mathbf{h}}$ is an open bounded subset with locally Lipschitz continuous boundary of $\mathbb{R}^{3}$ and the torso domain is occupied by $\Omega_{\mathbf{t}}$. We denote by $S$ the interface between both domains $\Omega_{\mathbf{h}}$ and $\Omega_{\mathrm{t}}$, by $\Gamma_{\text {ext }}$ the external boundary of $\Omega_{\mathrm{t}}$ and by $n$ the outward unit normal to $\Omega_{\mathrm{t}}$. Let $S^{+}$(resp. $S^{-}$) be the part of $S$ corresponding to the positive (resp. negative) direction of the normal $n$. We define the global domain $Q=\Omega \times(0, T)$ where $\Omega=\bar{\Omega}_{\mathbf{h}} \cup \Omega_{\mathbf{t}}$, ( see figure 1$)$.

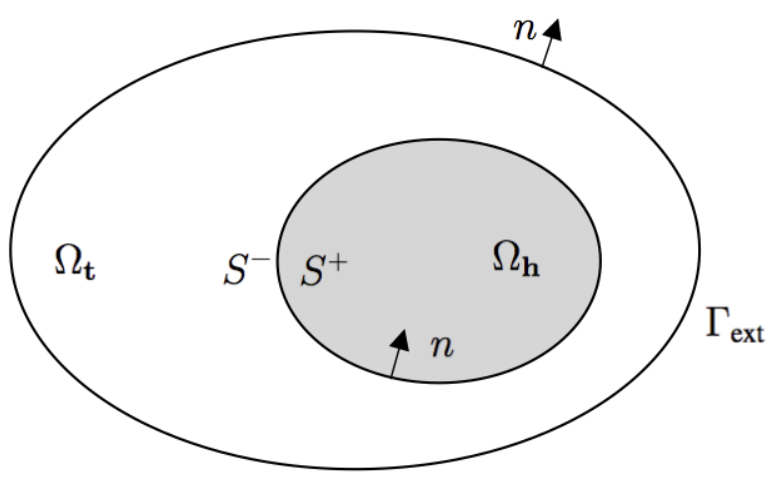

FIGURE 1. The heart and torso domains.

In order to describe the electrical activity of the heart, we use the monodomain model. This model allows to describe the propagation of the electrical wave in the myocardium. The monodomain equation is coupled to a set of dynamic system describing the physiology of the electrical activity at the cellular scale. The extracellular potential in the heart could be obtained by solving a Poisson equation in the heart domain. The following system is used in the literature to compute the extracellular potential in the heart. It is less complex than the bidomain model because the transmembrane potential is not coupled to the extracellular potential in the model.

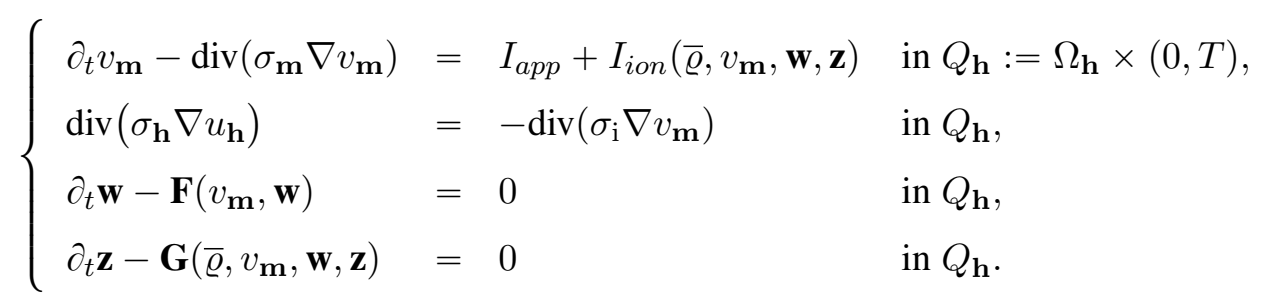

Here, the transmembrane potential $v_{\mathbf{m}}$ is defined as follows

$$
v_{\mathbf{m}}=u_{\mathbf{i}}-u_{\mathbf{h}}
$$

where $u_{\mathbf{i}}$ and $u_{\mathbf{h}}$ are the intra- and extra- cellular potentials. The $I_{a p p}$ is an external applied electrical current and $I_{i o n}$ is the ionic current across the membrane which is defined as follows

$$
I_{\text {ion }}(\bar{\varrho},, \mathbf{w}, \mathbf{z})=\sum_{i=1}^{N} \bar{\varrho}_{\mathbf{i}} y_{\mathbf{i}}(v) \prod_{j=1}^{k}\left(\tilde{w}_{l}\right)_{j}^{p_{j, i}}\left(v-E_{\mathbf{i}}(\mathbf{z})\right),
$$


where

$$
E_{\mathbf{i}}(\mathbf{z})=\bar{\gamma}_{\mathbf{i}} \log \left(\frac{z_{\mathbf{e}}}{z_{\mathbf{i}}}\right), \quad \mathbf{z}=\left(z_{1}, \ldots, z_{\mathbf{m}}\right)
$$

Here $\bar{\gamma}_{\mathbf{i}}$ is a constant and we denotes by $z_{\mathbf{i}}, i=1, \ldots, m$ and $z_{\mathbf{e}}$ the intra- and extracellular concentration. We define the evolution of the gating variables $\mathbf{w}:=\left(w_{1}, \ldots, w_{k}\right)$ and the ionc intracellular concentration variables $\mathbf{z}:=\left(z_{1}, \ldots, z_{\mathbf{m}}\right)$ by the following functions $F\left(v_{\mathbf{m}}, \mathbf{w}\right)$ and $G\left(\bar{\varrho}, v_{\mathbf{m}}, \mathbf{w}, \mathbf{z}\right)$ which are defined as follows

$$
\partial_{t} w_{j}=F_{j}\left(v_{\mathbf{m}}, w_{j}\right):=\alpha_{j}\left(v_{\mathbf{m}}\right)\left(1-w_{j}\right)-\beta_{j}\left(v_{\mathbf{m}}\right) w_{j}, \quad j=1, \ldots, k,
$$

where $\alpha_{j}$ and $\beta_{j}$ are a positive and smooth functions with $0 \leqslant w_{j} \leqslant 1$ and

$$
\partial_{t} z_{\mathbf{i}}=G_{\mathbf{i}}\left(\bar{\varrho}, v_{\mathbf{m}}, \mathbf{w}, \mathbf{z}\right):=-J_{\mathbf{i}}\left(\bar{\varrho}, v_{\mathbf{m}}, \mathbf{w}, \log z_{\mathbf{i}}\right)+H_{\mathbf{i}}\left(\bar{\varrho}, v_{\mathbf{m}}, \mathbf{w}, \mathbf{z}\right), \quad \forall i=1, \ldots, m,
$$

where

$$
J_{\mathbf{i}} \in C^{2}\left(\mathbb{R}_{+}^{*} \times \mathbb{R} \times \mathbb{R}^{k} \times \mathbb{R}\right), \quad 0<g_{*}(\mathbf{w}) \leqslant \frac{\partial J_{\mathbf{i}}}{\partial \tau}(\bar{\varrho}, v, \mathbf{w}, \tau) \leqslant g^{*}(\mathbf{w}), \quad\left|\frac{\partial J_{\mathbf{i}}}{\partial \tau}(\bar{\varrho}, v, \mathbf{w}, 0)\right| \leqslant L_{v}(\mathbf{w}),
$$

with $g_{*}, g^{*} L_{v}$ belong to $C^{1}\left(\mathbb{R}^{k}, \mathbb{R}_{+}\right)$and

$$
H_{\mathbf{i}} \in C^{2}\left(\mathbb{R}_{+}^{*} \times \mathbb{R} \times \mathbb{R}^{k} \times(0,+\infty)^{m}\right) \cap \operatorname{Lip}\left(\mathbb{R}_{+}^{*} \times \mathbb{R} \times[0,1]^{k} \times(0,+\infty)^{m}\right) .
$$

The system (1.2) is completed with the following condition on the interface boundary $\Sigma:=S \times(0, T)$

$$
\sigma_{\mathbf{m}} \nabla v_{\mathbf{m}} \cdot n=0 \text { on } \Sigma .
$$

Our mathematical model is based on the coupling of (1.2) with the following diffusion equation in $Q_{\mathbf{t}}=$ $\Omega_{\mathbf{t}} \times(0, T)$

$$
\operatorname{div}\left(\sigma_{\mathbf{t}} \nabla u_{\mathbf{t}}\right)=0 \text { in } Q_{\mathbf{t}},
$$

with the following condition on the external boundary $\Sigma_{\text {ext }}=\Gamma_{\text {ext }} \times(0, T)$ which is assumed to be isolated

$$
\sigma_{\mathbf{t}} \nabla u_{\mathbf{t}} \cdot n=0 \quad \text { on } \Sigma_{\text {ext }},
$$

where $u_{\mathbf{t}}$ and $\sigma_{\mathbf{t}}$ represent the torso potential and the conductivity tensor of the torso. In order to guarantee the continuity of the electrical potentials and currents from the heart to thorax, we need to introduce the following transmission conditions

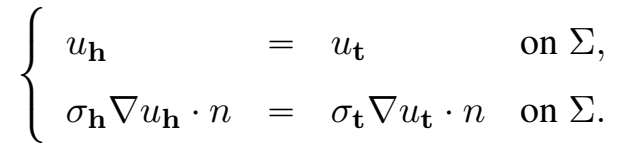

To sum up, from (1.2) - (1.10) - (1.11) - (1.12) and (1.13), we obtain the following the coupled heart-torso model

$$
\left\{\begin{array}{lll}
\partial_{t} v_{\mathbf{m}}-\operatorname{div}\left(\sigma_{\mathbf{m}} \nabla v_{\mathbf{m}}\right) & =I_{a p p}+I_{i o n}\left(\bar{\varrho}, v_{\mathbf{m}}, \mathbf{w}, \mathbf{z}\right) & \text { in } Q_{\mathbf{h}}, \\
\operatorname{div}\left(\sigma_{\mathbf{h}} \nabla u_{\mathbf{h}}\right) & =-\operatorname{div}\left(\sigma_{\mathrm{i}} \nabla v_{\mathbf{m}}\right) & \text { in } Q_{\mathbf{h}}, \\
\operatorname{div}\left(\sigma_{\mathbf{t}} \nabla u_{\mathbf{t}}\right) & =0 & \text { in } Q_{\mathbf{t}}, \\
\partial_{t} \mathbf{w}-\mathbf{F}\left(v_{\mathbf{m}}, \mathbf{w}\right) & =0 & \text { in } Q_{\mathbf{h}}, \\
\partial_{t} \mathbf{z}-\mathbf{G}\left(\bar{\varrho}, v_{\mathbf{m}}, \mathbf{w}, \mathbf{z}\right) & =0 & \text { in } Q_{\mathbf{h}},
\end{array}\right.
$$

with the following interface conditions

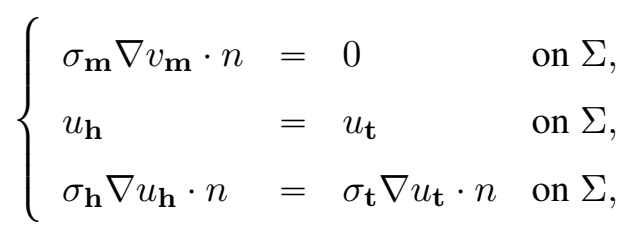

and the following external boundary condition 


$$
\sigma_{\mathbf{t}} \nabla u_{\mathbf{t}} \cdot n=0 \quad \text { on } \Sigma_{\text {ext }} .
$$

1.1. Properties of the solution. We start by examining the well-posedness and regularity of the solution for the heart-torso coupled system (1.14)-(1.15)-(1.16). We recall the following lemma on the unique existence of a solution for the heart-torso coupled system. The proof is based on [1], [39] and [40].

Lemma 1.1. Let $(v, u, \boldsymbol{w}, \boldsymbol{z})$ be the solution of the heart-torso coupled system (1.14)- (1.15)-(1.16), such that $I_{\text {app }} \in L^{p}\left(0, T ; L^{2}\left(\Omega_{\mathbf{h}}\right)\right) \cap H^{1}\left(0, T ; H^{2}\left(\Omega_{\mathbf{h}}\right)\right), p>4$, and the initial conditions $\left(v_{0}, \boldsymbol{w}_{0}, \boldsymbol{z}_{0}\right)$ satisfies the following regularities :

$$
v_{0} \in H^{4}\left(\Omega_{\mathbf{h}}\right), \quad \boldsymbol{w}_{0} \in H^{2}\left(\Omega_{\mathbf{h}}\right)^{k} \quad \text { and } \quad z_{0} \in H^{2}\left(\Omega_{\mathbf{h}}\right)^{m} .
$$

Then, we have

$$
\begin{aligned}
v_{\mathbf{m}} & \in H^{1}\left(0, T ; H^{3}\left(\Omega_{\mathbf{h}}\right)\right) \cap H^{2}\left(0, T ; H^{1}\left(\Omega_{\mathbf{h}}\right)\right), \\
\boldsymbol{w} & \in W^{1, \infty}\left(0, T ; H^{2}\left(\Omega_{\mathbf{h}}\right)\right)^{k} \cap H^{2}\left(0, T ; H^{1}\left(\Omega_{\mathbf{h}}\right)\right)^{k}, \\
\boldsymbol{z} & \in W^{1, \infty}\left(0, T ; H^{2}\left(\Omega_{\mathbf{h}}\right)\right)^{m} \cap H^{2}\left(0, T ; H^{1}\left(\Omega_{\mathbf{h}}\right)\right)^{m}, \\
u & \in H^{2}\left(0, T ; H^{1}(\Omega)\right) .
\end{aligned}
$$

Moreover if

we get

$$
\boldsymbol{w}_{0} \in H^{3}\left(\Omega_{\mathbf{h}}\right)^{k} \quad \text { and } \quad z_{0} \in H^{3}\left(\Omega_{\mathbf{h}}\right)^{m}
$$

$$
\begin{aligned}
& \boldsymbol{w} \in W^{1, \infty}\left(0, T ; H^{3}\left(\Omega_{\mathbf{h}}\right)\right)^{k} \hookrightarrow H^{1}\left(0, T ; H^{3}\left(\Omega_{\mathbf{h}}\right)\right)^{k}, \\
& \boldsymbol{z} \in W^{1, \infty}\left(0, T ; H^{3}\left(\Omega_{\mathbf{h}}\right)\right)^{m} \hookrightarrow H^{1}\left(0, T ; H^{3}\left(\Omega_{\mathbf{h}}\right)\right)^{m} .
\end{aligned}
$$

1.2. Inverse problem. The inverse problems are arousing more and more interest and can be formulated in different ways. This problem have attracted much attention to many researchers working in various applied fields. In general, there exist two types of formulations in the study of the inverse problems of determining coefficients or source terms of partial differential equations: the first type with an infinitely many measurements who we treat it by Dirichlet-to-Neumann-map and the other type with a finitely many measurements by means of Carleman estimates. In 1939, a Carleman estimate was first discovered by Carleman in [15] for proving the unique continuation for a two-dimensional elliptic equation. Thereafter, this tool has been an essential method to obtain unique continuous for partial differential operators with nonanalytical coefficients. In addition, Carleman estimates have also become the key ingredient for establishing stability results and this method was first introduced by Bukhgeim and Klibanov [12] which allows to prove the global uniqueness theorems. In other words, these theorems generally only require the regularity of the unknown coefficients. Later, this method was extended to nonlinear parabolic and elliptical equations in [26], [27] and [28]. By means of global Carleman estimates and for the first time in 1998 the Lipschitz stability of an inverse parabolic problem was established by Imanuvilov and Yamamoto in [24]. Since then, this type of inverse problems for parabolic equations has received a large amount of attention.

Our formulation of the inverse problem requires a finite number of observations. For this purpose, we follow the method proposed by Bukhgeim and Klibanov [12] that established the uniqueness for inverse problem of determining some coefficients which is based on a Carleman estimate. We refer also to Bellassoued [5, 6], Bellassoued and Yamamoto [7, 8], Benabdallah, Cristofol, Gaitan and Yamamoto [9], Bukhgeim [14], Bukhgeim, Cheng, Isakov and Yamamoto [13], Benabdallah, Gaitan, Le Rousseau [10] and Baudouin, Cerpa, Crpeau and Mercado [4]. Among others PDEs coefficient or source inverse problems, where Carleman estimates have been used, we can mention reactiondiffusion systems which are frequently used to model several physical applications, for example: in biology and medicine, emergence and growth of cancer.

In this paper, we study an inverse problem for the coupled heart torso system (1.14)-(1.15)-(1.16) modelling the electrical activity of the heart, in order to recover conductivities parameters from body surface 
measurements. In fact, the electric wave propagation in the heart can be formulated by a nonlinear reactiondiffusion system coupled to an ordinary differential equation system called the bidomain model $[30,18]$. In our case, we use the monodomain model which is a more simplified model of the bidomain model and on the other hand they are equivalent when the ratios of intracellular conductivity anisotropy are close to those in the extracellular domains. The bidomain and monodomain model equations $[38,25]$ are well established as the standard set of equations for the simulation of cardiac electrophysiology [36, 37, 35]. In the computational electrophysiology community, the monodomain model is the most used in order that is computationally much cheaper than the bidomain model. In this context, there are some works focused to the study of the cardiac parameters identifiability problem by using Carleman estimates $[1,2,29]$ and in the framework of the cardiac conductivities recovery problem by means of Carleman estimates, Aniseba, Bendahmane and Yuan in [3] obtains the stability results for the conductivities diffusion-coefficients and $\mathrm{Wu}$, Yan, Gao and Chen in [41] proved a Holder stability result for the inverse conductivities problem.

Let $\omega \subset \Omega_{\mathbf{h}}$ be a non-empty subdomain of $\Omega_{\mathbf{h}}$ and $\omega_{0} \subset \omega$, then there exists a weight function $\beta \in C^{0}(\bar{\Omega})$, $\beta_{\mathbf{i}}=\beta_{\mid \Omega_{\mathbf{i}}} \in C^{2}\left(\Omega_{\mathbf{i}}\right)$ with $\mathbf{i}=\mathbf{h}$ or $\mathbf{t}$ satisfied the following conditions :

$$
\begin{array}{rcc}
\beta=-1 & \text { on } & \Gamma_{\text {ext }}, \\
\partial_{n} \beta<0 & \text { on } & \Gamma_{\text {ext }}, \\
\beta=0 & \text { on } & S, \\
\partial_{n} \beta_{\mathbf{h}}>0, \partial_{n} \beta_{\mathbf{t}}>0 & \text { on } & S, \\
\sigma_{\mathbf{t}} \partial_{n} \beta_{\mathbf{t}}=\sigma_{\mathbf{h}} \partial_{n} \beta_{\mathbf{h}} & \text { on } & S,
\end{array}
$$

and

$$
|\nabla \beta|>0 \text { in } \bar{\Omega} \backslash \omega_{0}
$$

We denote $t \in(0, T)$ and $t_{0}=T / 2$.

Thereafter, we consider two sets of coefficients $\left(\sigma_{\mathbf{m}}, \sigma_{h}, \sigma_{\mathbf{i}}, \sigma_{\mathbf{t}}\right)$ and $\left(\hat{\sigma}_{\mathbf{m}}, \widehat{\sigma}_{h}, \widehat{\sigma}_{\mathbf{i}}, \widehat{\sigma}_{\mathbf{t}}\right)$ and the corresponding solutions $\left(u, v_{\mathbf{m}}, \mathbf{w}, \mathbf{z}\right)$ and $\left(\widehat{u}, \widehat{v}_{\mathbf{m}}, \widehat{\mathbf{w}}, \widehat{\mathbf{z}}\right)$ of (1.14)-(1.15)-(1.16). Let $\alpha \in C^{2}\left(\bar{\Omega}_{\mathbf{h}}\right)$ be a positive function $\alpha(x) \geqslant \alpha_{0}, x \in \bar{\Omega}_{\mathbf{h}}$, we define the following sets of admissible coefficients :

$$
\begin{gathered}
\mathcal{A}_{\alpha}^{\mathbf{h}}=\left\{\left(\sigma_{\mathbf{i}}, \sigma_{\mathbf{e}}\right) \in C^{2}\left(\bar{\Omega}_{\mathbf{h}}\right), \sigma_{\mathbf{i}} \geqslant c_{\mathbf{i}}>0, \sigma_{\mathbf{e}} \geqslant c_{\mathbf{e}}>0 \text { and } \sigma_{\mathbf{i}}=\alpha \sigma_{\mathbf{e}}\right\}, \\
\mathcal{A}^{\mathbf{t}}=\left\{\sigma_{\mathbf{t}} \in C^{2}\left(\bar{\Omega}_{\mathbf{t}}\right), \sigma_{\mathbf{t}} \geqslant c_{\mathbf{t}}>0\right\},
\end{gathered}
$$

for some positive constants $c_{\mathrm{i}}, c_{\mathrm{e}}$ and $c_{\mathrm{t}}$. In order to formulate our stability and uniqueness results of conductivities, we need to introduce the following assumptions :

Assumption (A.1). Let $\omega_{0} \subset \omega$. There exists a constants $c_{0}>0$ such that

$$
\inf _{x \in\left(\bar{\Omega} \mid \omega_{0}\right)}\left|\nabla \beta(x) \cdot \nabla \hat{d}\left(x, t_{0}\right)\right| \geqslant c_{0}, \quad \text { with } \widehat{d} \in\left\{\widehat{v}_{\mathbf{m}}, \widehat{u}_{\mathbf{h}}, \widehat{u}_{\mathbf{t}}\right\} .
$$

Assumption (A.2). There exists a constants $M>0$ such that

$$
\left\|\widehat{v}_{\mathbf{m}}\right\|_{W^{2, \infty}\left(0, T ; W^{2, \infty}\left(\Omega_{\mathbf{h}}\right)\right)}+\left\|\widehat{u}_{\mathbf{h}}\right\|_{W^{2, \infty}\left(0, T ; W^{2, \infty}\left(\Omega_{\mathbf{h}}\right)\right)}+\left\|\widehat{u}_{\mathbf{t}}\right\|_{W^{2, \infty}\left(0, T ; W^{2, \infty}\left(\Omega_{\mathbf{t}}\right)\right)} \leqslant M .
$$

Theorem 1.2. (Stability) We assume that (A.1) and (A.2) are satisfied. Then, there exists a positive constant $C>0$ depending on $\Omega, T$, and $M$, such that

$$
\begin{aligned}
& \left\|\sigma_{\mathbf{i}}-\widehat{\sigma}_{\mathbf{i}}\right\|_{H^{1}\left(\Omega_{\mathbf{h}}\right)}+\left\|\sigma_{\mathbf{e}}-\widehat{\sigma}_{\mathbf{e}}\right\|_{H^{1}\left(\Omega_{\mathbf{h}}\right)}+\left\|\sigma_{\mathbf{t}}-\widehat{\sigma}_{\mathbf{t}}\right\|_{H^{1}\left(\Omega_{\mathbf{t}}\right)} \leqslant C\left(\left\|\left(v_{\mathbf{m}}-\widehat{v}_{\mathbf{m}}\right)\left(\cdot, t_{0}\right)\right\|_{H^{2}\left(\Omega_{\mathbf{h}}\right)}\right. \\
& +\left\|\left(u_{\mathbf{t}}-\widehat{u}_{\mathbf{t}}\right)\left(\cdot, t_{0}\right)\right\|_{H^{2}\left(\Omega_{\mathbf{t}}\right)}+\left\|(\boldsymbol{w}-\widehat{\boldsymbol{w}})\left(\cdot, t_{0}\right)\right\|_{H^{1}\left(\Omega_{\mathbf{h}}\right)}+\left\|(\boldsymbol{z}-\widehat{\boldsymbol{z}})\left(\cdot, t_{0}\right)\right\|_{H^{1}\left(\Omega_{\mathbf{h}}\right)}+\left\|\sigma_{\mathbf{i}}-\widehat{\sigma}_{\mathbf{i}}\right\|_{H^{1}(\omega)} \\
& \quad+\left\|v_{\mathbf{m}}-\widehat{v}_{\mathbf{m}}\right\|_{H^{2}\left(0, T ; L^{2}(\omega)\right)}+\left\|u_{\mathbf{h}}-\widehat{u}_{\mathbf{h}}\right\|_{H^{2}\left(0, T ; L^{2}(\omega)\right)}+\left\|u_{\mathbf{t}}-\widehat{u}_{\mathbf{t}}\right\|_{\left.H^{2}\left(0, T ; H^{1}\left(\Gamma_{e x t}\right)\right)\right),}
\end{aligned}
$$

for any $\left(\sigma_{\mathbf{i}}, \sigma_{\mathbf{e}}\right),\left(\widehat{\sigma}_{\mathbf{i}}, \widehat{\sigma}_{\mathbf{e}}\right) \in \mathcal{A}_{\alpha}^{\mathbf{h}}, \sigma_{\mathbf{t}}, \widehat{\sigma}_{\mathbf{t}} \in \mathcal{A}^{\mathbf{t}}$ satisfying $\left(\partial^{\gamma} \sigma_{\mathbf{i}}, \partial^{\gamma} \sigma_{\mathbf{e}}\right)=\left(\partial^{\gamma} \widehat{\sigma}_{\mathbf{i}}, \partial^{\gamma} \widehat{\sigma}_{\mathbf{e}}\right)$ on $S,|\gamma| \leqslant 1$ and $\sigma_{\mathbf{t}} \geqslant \sigma_{\mathbf{h}}$ on $S$. 
As a consequence, we can drive the following uniqueness result

Corollary 1.3. (Uniqueness) Let us consider the same assumptions in Theorem 1.2 and let $\left(v_{\mathbf{m}}, u_{\mathbf{t}}, \boldsymbol{w}, \boldsymbol{z}\right)=$ $\left(\widehat{v}_{\mathbf{m}}, \widehat{u}_{\mathbf{t}}, \widehat{\boldsymbol{w}}, \widehat{\boldsymbol{z}}\right)$ at a fixed time $t_{0},\left(v_{\mathbf{m}}, u_{\mathbf{h}}\right)=\left(\widehat{v}_{\mathbf{m}}, \widehat{u}_{\mathbf{h}}\right)$ in $\omega \times(0, T), u_{\mathbf{t}}=\widehat{u}_{\mathbf{t}}$ in the external boundary $\Sigma_{\text {ext }}$ and $\sigma_{\mathbf{i}}=\hat{\sigma}_{\mathbf{i}}$ in $\omega$. Then, we have the following uniqueness result

$$
\left(\sigma_{\mathbf{i}}, \sigma_{\mathbf{e}}\right)=\left(\hat{\sigma}_{\mathbf{i}}, \hat{\sigma}_{\mathbf{e}}\right) \text { in } \Omega_{\mathbf{h}}, \quad \text { and } \sigma_{\mathbf{t}}=\hat{\sigma}_{\mathbf{t}} \text { in } \Omega_{\mathbf{t}} .
$$

The assumptions (A.1) and (A.2) are commonly used in the study of inverse problems, we can cite [1,9]. Assumption (A.1) can be satisfied for a suitable smooth initial data and to sufficiently small. If $T$ is small enough or the initial data are sufficiently smooth, then (A.2) is satisfied.

The remainder of the paper is organized as follows : In section 2, we prove a global Carleman inequality for the coupled heart-torso model with a singular weight function which is the key ingredient to establish the stability estimates for some coefficients appearing in our model given by (1.14)-(1.15)-(1.16). By means of this estimate in section 3, following the Bukhgeim-Klibanov method we prove the stability result for the inverse conductivities problem. In section 4 , we prove the stability estimate of conductance parameters.

\section{CARLemAn Estimate}

In this section, we will establish a Carleman estimate for the monodomain coupled system (1.14)-(1.15)(1.16). In order to get our Carleman estimate, we need a weight function with special properties for parabolic equations so called singular weight function and we refer to Fursikov and Imanuvilov [22], Imanuvilov [20], Imanuvilov and Yamamoto [24], Doubova, Osses and Puel [19].

2.1. Weight function. Let us define the following weight function : Let $\omega \subset \Omega_{\mathbf{h}}$ and for a non-empty subdomain $\omega_{0} \subset \omega$, there exists a function $\beta \in C^{0}(\bar{\Omega}), \beta_{\mathbf{i}}=\beta_{\mid \Omega_{\mathbf{i}}} \in C^{2}\left(\Omega_{\mathbf{i}}\right), \mathbf{i}=\mathbf{h}$ or $\mathbf{t}$, such that we have the following conditions

$$
\begin{array}{rcc}
\beta=-1 & \text { on } & \Gamma_{\text {ext }}, \\
\partial_{n} \beta<0 & \text { on } & \Gamma_{\text {ext }}, \\
\beta=0 & \text { on } & S, \\
\partial_{n} \beta_{\mathbf{h}}>0, \partial_{n} \beta_{\mathbf{t}}>0 & \text { on } & S, \\
\sigma_{\mathbf{t}} \partial_{n} \beta_{\mathbf{t}}=\sigma_{\mathbf{h}} \partial_{n} \beta_{\mathbf{h}} & \text { on } & S .
\end{array}
$$

Moreover, we have

$$
|\nabla \beta|>0 \text { in } \bar{\Omega} \backslash \omega_{0} .
$$

As for the existence of $\beta$, see [19] Lemma 3.1. The jump of the function $\beta$ on $S$ satisfy

$$
\left[\partial_{n} \beta\right]_{S}=\partial_{n} \beta_{\mathbf{h}}-\partial_{n} \beta_{\mathbf{t}}=\left(\frac{\sigma_{\mathbf{t}}}{\sigma_{\mathbf{h}}}-1\right) \partial_{n} \beta_{\mathbf{t}} \geqslant 0,
$$

for $\sigma_{\mathbf{t}}>\sigma_{\mathbf{h}}$ on $S$. We will now use the function $\beta$ given by (2.1) to build new weight function. Let $\lambda>0$ and $\ell(t)=t(T-t), t \in(0, T)$ we introduce the following weight functions :

$$
\varphi(x, t)=\frac{e^{\lambda \beta(x)}}{\ell(t)}, \quad \eta(x, t)=\frac{e^{2 \lambda\|\beta\|_{\infty}}-e^{\lambda \beta(x)}}{\ell(t)} .
$$

Notice that

$$
\nabla \varphi(x, t)=\lambda \varphi \nabla \beta, \quad \nabla \eta(x, t)=-\lambda \varphi \nabla \beta .
$$

We use usual functions space, $H^{k}\left(Q_{\mathbf{h}}\right)$, and

$$
H^{1,2}\left(Q_{\mathbf{h}}\right)=H^{1}\left(0, T ; L^{2}\left(\Omega_{\mathbf{h}}\right)\right) \cap L^{2}\left(0, T ; H^{2}\left(\Omega_{\mathbf{h}}\right)\right) .
$$


2.2. Carleman estimate for the transmembarne potential. Here, we give Carleman estimate for the transmembarne potential.

We consider the following boundary value problem for parabolic equation :

$$
\begin{cases}\partial_{t} v_{\mathbf{m}}-\operatorname{div}\left(\sigma_{\mathbf{m}} \nabla v_{\mathbf{m}}\right)=g & \text { in } Q_{\mathbf{h}}, \\ \sigma_{\mathbf{m}} \nabla v_{\mathbf{m}} \cdot n=0 & \text { on } \Sigma,\end{cases}
$$

where $g \in L^{2}\left(Q_{\mathbf{h}}\right)$. Then, we recall the following parabolic Carleman estimate with Neumann boundary condition proved in Lemma 2.2 in [24], (see also [21]).

Lemma 2.1. Let $p \in \mathbb{N}$. Then, there exists $\lambda_{0}>0$ such that for any $\lambda \geqslant \lambda_{0}$ there exist $s_{0}:=s_{0}(\lambda)>0$ and $C=C_{\lambda}>0$ such that the following estimate holds

$$
\begin{aligned}
& \int_{Q_{\mathbf{h}}}\left(( s \varphi ) ^ { p - 1 } \left(\left|\partial_{t} v_{\mathbf{m}}\right|^{2}+\mid\right.\right.\left.\left.\left.\operatorname{div}\left(\sigma_{\mathbf{m}} \nabla v_{\mathbf{m}}\right)\right|^{2}\right)+(s \varphi)^{p+3}\left|v_{\mathbf{m}}\right|^{2}+(s \varphi)^{p+1}\left|\nabla v_{\mathbf{m}}\right|^{2}\right) e^{-2 s \eta} d x d t \\
& \leqslant C\left(\int_{Q_{\mathbf{h}}}(s \varphi)^{p}|g|^{2} e^{-2 s \eta} d x d t+\int_{\omega \times(0, T)}(s \varphi)^{p+3}\left|v_{\mathbf{m}}\right|^{2} e^{-2 s \eta} d x d t\right)
\end{aligned}
$$

for any $s>s_{0}$ and $v_{\mathbf{m}} \in H^{1,2}\left(Q_{\mathbf{h}}\right)$ satisfies (2.5).

2.3. Carleman estimate for the transmission elliptic problem. In this section, we derive a global Carleman estimate for a solution of the elliptic transmission system. In $Q=Q_{\mathbf{h}} \cup Q_{\mathbf{t}}$, we consider the following system :

$$
\begin{cases}\operatorname{div}\left(\sigma_{\mathbf{h}} \nabla u_{\mathbf{h}}\right)=F_{\mathbf{h}} & \text { in } Q_{\mathbf{h}}, \\ \operatorname{div}\left(\sigma_{\mathbf{t}} \nabla u_{\mathbf{t}}\right)=F_{\mathbf{t}} & \text { in } Q_{\mathbf{t}},\end{cases}
$$

where $F_{\mathbf{h}} \in L^{2}\left(Q_{\mathbf{h}}\right)$ and $F_{\mathbf{t}} \in L^{2}\left(Q_{\mathbf{t}}\right)$. A perfect electric heart-torso coupling, across the interface $\Sigma$, given by the following transmission conditions :

$$
\begin{cases}u_{\mathbf{h}}=u_{\mathbf{t}} & \text { on } \Sigma, \\ \sigma_{\mathbf{h}} \nabla u_{\mathbf{h}} \cdot n=\sigma_{\mathbf{t}} \nabla u_{\mathbf{t}} \cdot n & \text { on } \Sigma,\end{cases}
$$

and the following exterior boundary condition

$$
\sigma_{\mathbf{t}} \partial_{n} u_{\mathbf{t}}=0 \quad \text { on } \quad \Sigma_{\text {ext }}
$$

We denote by $u=u_{\mathbf{h}} \chi_{\Omega_{\mathbf{h}}}+u_{\mathbf{t}} \chi_{\Omega_{\mathbf{t}}}$ the solution of (2.7)-(2.8)-(2.9) and $F=F_{\mathbf{h}} \chi_{\Omega_{\mathbf{h}}}+F_{\mathbf{h}} \chi_{\Omega_{\mathbf{h}}}$. Moreover, we assume that $\sigma_{\mathbf{t}}>\sigma_{\mathbf{h}}$ on $S$. Then, we have the following Carleman estimate :

Theorem 2.2. Under the previous assumptions, there exists $\lambda_{0}>0$ such that for any $\lambda \geqslant \lambda_{0}$ there exist $s_{0}:=s_{0}(\lambda)>0$ and $C=C_{\lambda}>0$ such that the solution of (2.7), (2.8) and (2.9) satisfies

$$
\begin{aligned}
\int_{Q}\left((s \varphi)^{3}|u|^{2}+(s \varphi)|\nabla u|^{2}\right) e^{-2 s \eta} d x d t \leqslant C\left(\int_{Q}|F|^{2} e^{-2 s \eta} d x d t\right. \\
\left.+\int_{\omega \times(0, T)}(s \varphi)^{3}\left|u_{\mathbf{h}}\right|^{2} e^{-2 s \eta} d x d t+\int_{\Sigma_{\text {ext }}}(s \varphi)\left|\nabla_{\tau} u_{\mathbf{t}}\right|^{2} e^{-2 s \eta} d x d t\right),
\end{aligned}
$$

for any $s>s_{0}$.

Proof. Let us introduce the following function $z(x, t)=e^{-s \eta} u(x, t)$ with $s>0$, where

$$
z=z_{\mathbf{h}} \chi_{\Omega_{\mathbf{h}}}+z_{\mathbf{t}} \chi_{\Omega_{\mathbf{t}}} \quad \text { and } \quad \eta=\eta_{\mathbf{h}} \chi_{\Omega_{\mathbf{h}}}+\eta_{\mathbf{t}} \chi_{\Omega_{\mathbf{t}}} .
$$

We denote $\sigma=\sigma_{\mathbf{h}} \chi_{\Omega_{\mathbf{h}}}+\sigma_{\mathbf{t}} \chi_{\Omega_{\mathbf{t}}}$. The standard approach to the form (2.10) of Carleman estimate starts from the observation

$$
e^{-s \eta} \operatorname{div}(\sigma \nabla u)=e^{-s \eta} \operatorname{div}\left(\sigma \nabla\left(e^{s \eta} z\right)\right)=e^{-s \eta} F:=P_{s}(z) .
$$


After computations, we split $P_{s}(z)$ into three terms as follows

$$
P_{s}(z)=L_{1, s}(z)+L_{2, s}(z)+R_{s}(z),
$$

where

$$
L_{1, s}(z)=\operatorname{div}(\sigma \nabla z)+s^{2} \lambda^{2} \varphi^{2} \sigma|\nabla \beta|^{2} z, \quad L_{2, s}(z)=-2 s \lambda \varphi \sigma(\nabla \beta \cdot \nabla z)-2 s \lambda^{2} \sigma \varphi z|\nabla \beta|^{2},
$$
and

$$
R_{s}(z)=s \lambda^{2} \sigma \varphi z|\nabla \beta|^{2}-s \lambda \varphi \operatorname{div}(\sigma \nabla \beta) z .
$$

With the previous notations, we get

$$
L_{1, s}(z)+L_{2, s}(z)=F_{s}(z),
$$

where $F_{s}(z)=e^{-s \eta} F-R_{s}(z)$. Applying the norm $L^{2}(Q)$ to (2.12), we get

$$
\left\|L_{1, s}(z)\right\|_{2}^{2}+\left\|L_{2, s}(z)\right\|_{2}^{2}+2\left(L_{1, s}(z), L_{2, s}(z)\right)=\left\|F_{s}(z)\right\|_{2}^{2} .
$$

Next we calculate $\left(L_{1, s}(z), L_{2, s}(z)\right)$ to look for the lower bound :

$$
\left(L_{1, s}(z), L_{2, s}(z)\right)=J_{11}+J_{12}+J_{21}+J_{22} .
$$

Let us consider the first term, we have

$$
\begin{aligned}
J_{11}= & -2 s \lambda \int_{Q} \sigma \varphi \operatorname{div}(\sigma \nabla z)(\nabla \beta \cdot \nabla z) d x d t \\
= & 2 s \lambda \int_{Q} \sigma \nabla z \cdot \nabla(\sigma \varphi \nabla \beta \cdot \nabla z) d x d t+2 s \lambda \int_{\Sigma^{+}} \varphi \sigma_{\mathbf{h}}^{2}\left(\nabla \beta_{\mathbf{h}} \cdot \nabla z_{\mathbf{h}}\right)\left(\partial_{n} z_{\mathbf{h}}\right) d x d t \\
& -2 s \lambda \int_{\Sigma^{-}} \varphi \sigma_{\mathbf{t}}^{2}\left(\nabla \beta_{\mathbf{t}} \cdot \nabla z_{\mathbf{t}}\right)\left(\partial_{n} z_{\mathbf{t}}\right) d x d t-2 s \lambda \int_{\Sigma_{\text {ext }}} \varphi \sigma_{\mathbf{t}}^{2}\left(\nabla \beta_{\mathbf{t}} \cdot \nabla z_{\mathbf{t}}\right)\left(\partial_{n} z_{\mathbf{t}}\right) d x d t .
\end{aligned}
$$

Then, separately we will calculate the first integrals in (2.18) in $Q_{\mathbf{t}}$ and in $Q_{\mathbf{h}}$.

$$
\begin{aligned}
J_{11}^{\mathbf{t}}=2 s \lambda^{2} \int_{Q_{\mathbf{t}}} \varphi \sigma_{\mathbf{t}}^{2}\left|\nabla z_{\mathbf{t}} \cdot \nabla \beta_{\mathbf{t}}\right|^{2} d x d t+2 s \lambda \sum_{j, k=1}^{n} \int_{Q_{\mathbf{t}}} \varphi \sigma_{\mathbf{t}} \partial_{x_{j}} z_{\mathbf{t}} \partial_{x_{j}}\left(\sigma_{\mathbf{t}} \partial_{x_{k}} \beta_{\mathbf{t}}\right) \partial_{x_{k}} z_{\mathbf{t}} d x d t \\
\quad+s \lambda \int_{Q_{\mathbf{t}}} \varphi \sigma_{\mathbf{t}}^{2} \nabla \beta_{\mathbf{t}} \cdot \nabla\left(\left|\nabla z_{\mathbf{t}}\right|^{2}\right) d x d t .
\end{aligned}
$$

Simplifying the expression of the last term,

$$
\begin{array}{rl}
J_{11}^{\mathbf{t}}=2 & s \lambda^{2} \int_{Q_{\mathbf{t}}} \varphi \sigma_{\mathbf{t}}^{2}\left|\nabla z_{\mathbf{t}} \cdot \nabla \beta_{\mathbf{t}}\right|^{2} d x d t+2 s \lambda \sum_{j, k=1}^{n} \int_{Q_{\mathbf{t}}} \varphi \sigma_{\mathbf{t}} \partial_{x_{j}} z_{\mathbf{t}} \partial_{x_{j}}\left(\sigma_{\mathbf{t}} \partial_{x_{k}} \beta_{\mathbf{t}}\right) \partial_{x_{k}} z_{\mathbf{t}} d x d t \\
& -s \lambda^{2} \int_{Q_{\mathbf{t}}} \varphi\left|\sigma_{\mathbf{t}} \nabla \beta_{\mathbf{t}}\right|^{2}\left|\nabla z_{\mathbf{t}}\right|^{2} d x d t-s \lambda \int_{Q_{\mathbf{t}}} \varphi\left|\nabla z_{\mathbf{t}}\right|^{2} \operatorname{div}\left(\sigma_{\mathbf{t}}^{2} \nabla \beta_{\mathbf{t}}\right) d x d t \\
& +s \lambda \int_{\Sigma^{-}} \varphi \sigma_{\mathbf{t}}^{2}\left(\partial_{n} \beta_{\mathbf{t}}\right)\left|\nabla z_{\mathbf{t}}\right|^{2} d x d t+s \lambda \int_{\Sigma_{\text {ext }}} \varphi \sigma_{\mathbf{t}}^{2}\left(\partial_{n} \beta_{\mathbf{t}}\right)\left|\nabla z_{\mathbf{t}}\right|^{2} d x d t .
\end{array}
$$

On the other hand, we have

$$
\begin{aligned}
J_{11}^{\mathbf{h}}= & 2 s \lambda^{2} \int_{Q_{\mathbf{h}}} \varphi \sigma_{\mathbf{h}}^{2}\left|\nabla z_{\mathbf{h}} \cdot \nabla \beta_{\mathbf{h}}\right|^{2} d x d t+2 s \lambda \sum_{j, k=1} \int_{Q_{\mathbf{h}}} \varphi \sigma_{\mathbf{h}} \partial_{x_{j}} z_{\mathbf{h}} \partial_{x_{j}}\left(\sigma_{\mathbf{h}} \partial_{x_{k}} \beta_{\mathbf{h}}\right) \partial_{x_{k}} z_{\mathbf{h}} d x d t \\
& -s \lambda^{2} \int_{Q_{\mathbf{h}}} \varphi\left|\sigma_{\mathbf{h}} \nabla \beta_{\mathbf{h}}\right|^{2}\left|\nabla z_{\mathbf{h}}\right|^{2} d x d t-s \lambda \int_{Q_{\mathbf{h}}} \varphi \operatorname{div}\left(\sigma_{\mathbf{h}}^{2} \nabla \beta_{\mathbf{h}}\right)\left|\nabla z_{\mathbf{h}}\right|^{2} d x d t \\
& -s \lambda \int_{\Sigma^{+}} \varphi \sigma_{\mathbf{h}}^{2}\left(\partial_{n} \beta_{\mathbf{h}}\right)\left|\nabla z_{\mathbf{h}}\right|^{2} d x d t .
\end{aligned}
$$


ANALYSIS OF THE HEART-TORSO CONDUCTIVITY PARAMETERS RECOVERY INVERSE PROBLEM IN CARDIAC ELECTROPHYSIOLOGY ECG

We can write $J_{11}$ as follows

$$
\begin{aligned}
J_{11}= & 2 s \lambda^{2} \int_{Q} \varphi \sigma^{2}|\nabla z \cdot \nabla \beta|^{2} d x d t+2 s \lambda \sum_{j, k=1}^{n} \int_{Q} \varphi \sigma\left(\partial_{x_{j}} z\right) \partial_{x_{j}}\left(\sigma \partial_{x_{k}} \beta\right)\left(\partial_{x_{k}} z\right) d x d t \\
& -s \lambda^{2} \int_{Q} \varphi|\sigma \nabla \beta|^{2}|\nabla z|^{2} d x d t-s \lambda \int_{Q} \varphi \operatorname{div}\left(\sigma^{2} \nabla \beta\right)|\nabla z|^{2} d x d t+\mathcal{B}(z),
\end{aligned}
$$

where the boundary term $\mathcal{B}(z)$ is given by

$$
\begin{aligned}
\mathcal{B}(z) & =2 s \lambda \int_{\Sigma^{+}} \varphi \sigma_{\mathbf{h}}^{2}\left(\partial_{n} z_{\mathbf{h}}\right)\left(\nabla \beta_{\mathbf{h}} \cdot \nabla z_{\mathbf{h}}\right) d x d t-s \lambda \int_{\Sigma^{+}} \varphi \sigma_{\mathbf{h}}^{2}\left(\partial_{n} \beta_{\mathbf{h}}\right)\left|\nabla z_{\mathbf{h}}\right|^{2} d x d t \\
& -2 s \lambda \int_{\Sigma^{-}} \varphi \sigma_{\mathbf{t}}^{2}\left(\partial_{n} z_{\mathbf{t}}\right)\left(\nabla \beta_{\mathbf{t}} \cdot \nabla z_{\mathbf{t}}\right) d x d t+s \lambda \int_{\Sigma^{-}} \varphi \sigma_{\mathbf{t}}^{2}\left(\partial_{n} \beta_{\mathbf{t}}\right)\left|\nabla z_{\mathbf{t}}\right|^{2} d x d t \\
& -2 s \lambda \int_{\Sigma_{\mathrm{ext}}} \varphi \sigma_{\mathbf{t}}^{2}\left(\nabla \beta_{\mathbf{t}} \cdot \nabla z_{\mathbf{t}}\right)\left(\partial_{n} z_{\mathbf{t}}\right) d x d t+s \lambda \int_{\Sigma_{\text {ext }}} \varphi \sigma_{\mathbf{t}}^{2}\left(\partial_{n} \beta_{\mathbf{t}}\right)\left|\nabla z_{\mathbf{t}}\right|^{2} d x d t
\end{aligned}
$$

Therefore,

$$
\begin{aligned}
& \mathcal{B}(z)=s \lambda \int_{\Sigma^{+}} \varphi \partial_{n} \beta_{\mathbf{h}}\left|\sigma_{\mathbf{h}} \partial_{n} z_{\mathbf{h}}\right|^{2} d x d t-s \lambda \int_{\Sigma^{-}} \varphi \partial_{n} \beta_{\mathbf{t}}\left|\sigma_{\mathbf{t}} \partial_{n} z_{\mathbf{t}}\right|^{2} d x d t \\
& -s \lambda \int_{\Sigma^{+}} \varphi \sigma_{\mathbf{h}}\left(\sigma_{\mathbf{h}} \partial_{n} \beta_{\mathbf{h}}\right)\left|\nabla_{\tau} z_{\mathbf{h}}\right|^{2} d x d t+s \lambda \int_{\Sigma^{-}} \varphi \sigma_{\mathbf{t}}\left(\sigma_{\mathbf{t}} \partial_{n} \beta_{\mathbf{t}}\right)\left|\nabla_{\tau} z_{\mathbf{t}}\right|^{2} d x d t \\
& -s \lambda \int_{\Sigma_{\text {ext }}} \varphi \sigma_{\mathbf{t}}^{2}\left(\partial_{n} \beta_{\mathbf{t}}\right)\left|\partial_{n} z_{\mathbf{t}}\right|^{2} d x d t+s \lambda \int_{\Sigma_{\text {ext }}} \varphi \sigma_{\mathbf{t}}^{2}\left(\partial_{n} \beta_{\mathbf{t}}\right)\left|\nabla_{\tau} z_{\mathbf{t}}\right|^{2} d x d t .
\end{aligned}
$$

here, we have used

$$
\nabla z=(\nabla z \cdot n) n+\nabla_{\tau} z, \quad \nabla \beta=(\nabla \beta \cdot n) n+\nabla_{\tau} \beta
$$

and the fact that $\nabla_{\tau} \beta=0$ on $\Sigma$. Thereafter, by using the fact that $\sigma_{\mathbf{t}} \partial_{n} u_{\mathbf{t}}=0$ on $\Sigma_{\text {ext }}$, we can see that

$$
\sigma_{\mathbf{t}} \partial_{n} z_{\mathbf{t}}=s \lambda \sigma_{\mathbf{t}} \varphi\left(\partial_{n} \beta_{\mathbf{t}}\right) z_{\mathbf{t}}
$$

and we apply the following transmission conditions on $S$,

$$
\begin{array}{lll}
\sigma_{\mathbf{t}} \partial_{n} \beta_{\mathbf{t}} & =\sigma_{\mathbf{h}} \partial_{n} \beta_{\mathbf{h}} & \text { on } S, \\
z_{\mathbf{h}} & =z_{\mathbf{t}} & \text { on } S, \\
\sigma_{\mathbf{h}} \partial_{n} z_{\mathbf{h}} & =\sigma_{\mathbf{t}} \partial_{n} z_{\mathbf{t}} & \text { on } S,
\end{array}
$$

we obtain

$$
\begin{aligned}
J_{11} & =2 s \lambda^{2} \int_{Q} \varphi \sigma^{2}|\nabla z \cdot \nabla \beta|^{2} d x d t-s \lambda^{2} \int_{Q} \varphi|\sigma \nabla \beta|^{2}|\nabla z|^{2} d x d t \\
& +s \lambda \int_{\Sigma} \varphi\left|\sigma \partial_{n} z\right|^{2}\left[\partial_{n} \beta\right]_{S} d x d t-s \lambda \int_{\Sigma} \varphi\left(\sigma \partial_{n} \beta\right)\left|\nabla_{\tau} z\right|^{2}[\sigma]_{S} d x d t \\
& -s^{3} \lambda^{3} \int_{\Sigma_{\mathrm{ext}}} \varphi^{3}\left|\sigma_{\mathbf{t}}\left(\partial_{n} \beta_{\mathbf{t}}\right)\right|^{2}\left(\partial_{n} \beta_{\mathbf{t}}\right)\left|z_{\mathbf{t}}\right|^{2} d x d t+Y_{1},
\end{aligned}
$$

where the remainder term $Y_{1}$ is given by

$$
\begin{aligned}
Y_{1}=2 s \lambda & \sum_{j, k=1}^{n} \int_{Q} \varphi \sigma\left(\partial_{x_{j}} z\right) \partial_{x_{j}}\left(\sigma \partial_{x_{k}} \beta\right)\left(\partial_{x_{k}} z\right) d x d t-s \lambda \int_{Q} \varphi \operatorname{div}\left(\sigma^{2} \nabla \beta\right)|\nabla z|^{2} d x d t \\
& +s \lambda \int_{\Sigma_{\mathrm{ext}}} \varphi\left(\partial_{n} \beta_{\mathbf{t}}\right)\left|\sigma_{\mathbf{t}} \nabla_{\tau} z_{\mathbf{t}}\right|^{2} d x d t
\end{aligned}
$$


We compute now the second term $J_{12}$, we have

$$
\begin{aligned}
J_{12}= & -2 s \lambda^{2} \int_{Q} \varphi z \sigma|\nabla \beta|^{2} \operatorname{div}(\sigma \nabla z) d x d t \\
= & 2 s \lambda^{2} \int_{Q} \sigma \nabla z \cdot \nabla\left(\varphi \sigma|\nabla \beta|^{2} z\right) d x d t+2 s \lambda^{2} \int_{\Sigma^{+}} \varphi z_{\mathbf{h}} \sigma_{\mathbf{h}}^{2}\left|\nabla \beta_{\mathbf{h}}\right|^{2}\left(\partial_{n} z_{\mathbf{h}}\right) d x d t \\
& -2 s \lambda^{2} \int_{\Sigma^{-}} \varphi z_{\mathbf{t}} \sigma_{\mathbf{t}}^{2}\left|\nabla \beta_{\mathbf{t}}\right|^{2}\left(\partial_{n} z_{\mathbf{t}}\right) d x d t-2 s \lambda^{2} \int_{\Sigma_{\text {ext }}} \varphi z_{\mathbf{t}} \sigma_{\mathbf{t}}^{2}\left|\nabla \beta_{\mathbf{t}}\right|^{2}\left(\partial_{n} z_{\mathbf{t}}\right) d x d t .
\end{aligned}
$$

Then, using again (2.18) and (2.21), we get

$$
J_{12}=2 s \lambda^{2} \int_{Q} \varphi|\sigma \nabla \beta|^{2}|\nabla z|^{2} d x d t-2 s^{2} \lambda^{3} \int_{\Sigma_{\mathrm{ext}}} \varphi^{2}\left|\sigma_{\mathbf{t}} \partial_{n} \beta_{\mathbf{t}}\right|^{2}\left(\partial_{n} \beta_{\mathbf{t}}\right)\left|z_{\mathbf{t}}\right|^{2} d x d t+Y_{2},
$$

where

$$
\begin{aligned}
Y_{2}= & 2 s \lambda^{2} \int_{Q} \varphi \sigma z \nabla z \cdot \nabla\left(\sigma|\nabla \beta|^{2}\right) d x d t+2 s \lambda^{3} \int_{Q} \sigma^{2} \varphi z(\nabla z \cdot \nabla \beta)|\nabla \beta|^{2} d x d t \\
& +2 s \lambda^{2} \int_{\Sigma} \varphi z\left(\sigma \partial_{n} z\right)\left(\sigma \partial_{n} \beta\right)\left[\partial_{n} \beta\right]_{S} d x d t .
\end{aligned}
$$

After computations, we also see that

$$
\begin{aligned}
J_{21}= & -2 s^{3} \lambda^{3} \int_{Q} \varphi^{3} \sigma^{2}|\nabla \beta|^{2} z(\nabla \beta \cdot \nabla z) d x d t \\
= & -s^{3} \lambda^{3} \int_{Q} \varphi^{3} \sigma^{2}|\nabla \beta|^{2} \nabla \beta \cdot \nabla\left(|z|^{2}\right) d x d t \\
= & 3 s^{3} \lambda^{4} \int_{Q} \varphi^{3} \sigma^{2}|\nabla \beta|^{4}|z|^{2} d x d t+s^{3} \lambda^{3} \int_{\Sigma} \varphi^{3}\left|\sigma \partial_{n} \beta\right|^{2}\left[\partial_{n} \beta\right]_{S}|z|^{2} d x d t \\
& \quad-s^{3} \lambda^{3} \int_{\Sigma_{\text {ext }}} \varphi^{3}\left|\sigma_{\mathbf{t}} \partial_{n} \beta_{\mathbf{t}}\right|^{2}\left|z_{\mathbf{t}}\right|^{2}\left(\partial_{n} \beta_{\mathbf{t}}\right) d x d t+Y_{3},
\end{aligned}
$$

where $Y_{3}$ is given by

$$
Y_{3}=s^{3} \lambda^{3} \int_{Q} \varphi^{3}|z|^{2} \operatorname{div}\left(\sigma^{2}|\nabla \beta|^{2} \nabla \beta\right) d x d t
$$

Finally, we have

$$
J_{22}=-2 s^{3} \lambda^{4} \int_{Q} \varphi^{3}|z|^{2} \sigma^{2}|\nabla \beta|^{4} d x d t .
$$

By summing (2.23), (2.25), (2.27) and (2.29), we get

$$
\begin{aligned}
\left(L_{1, s}(z), L_{2, s}(z)\right) & =s^{3} \lambda^{4} \int_{Q} \varphi^{3}|z|^{2} \sigma^{2}|\nabla \beta|^{4} d x d t+2 s \lambda^{2} \int_{Q} \varphi \sigma^{2}|\nabla z \cdot \nabla \beta|^{2} d x d t \\
& +s \lambda^{2} \int_{Q} \varphi|\sigma \nabla \beta|^{2}|\nabla z|^{2} d x d t-2 s^{3} \lambda^{3} \int_{\Sigma_{\mathrm{ext}}} \varphi^{3}\left|\sigma_{\mathbf{t}} \partial_{n} \beta_{\mathbf{t}}\right|^{2} \partial_{n} \beta_{\mathbf{t}}\left|z_{\mathbf{t}}\right|^{2} d x d t \\
& -2 s^{2} \lambda^{3} \int_{\Sigma_{\mathrm{ext}}} \varphi^{2}\left|\sigma_{\mathbf{t}} \partial_{n} \beta_{\mathbf{t}}\right|^{2} \partial_{n} \beta_{\mathbf{t}}\left|z_{\mathbf{t}}\right|^{2} d x d t+s \lambda \int_{\Sigma} \varphi\left|\sigma \partial_{n} z\right|^{2}\left[\partial_{n} \beta\right]_{S} d x d t \\
& -s \lambda \int_{\Sigma} \varphi\left(\sigma \partial_{n} \beta\right)\left|\nabla_{\tau} z\right|^{2}[\sigma]_{S} d x d t+s^{3} \lambda^{3} \int_{\Sigma} \varphi^{3}\left|\sigma \partial_{n} \beta\right|^{2}\left[\partial_{n} \beta\right]_{S}|z|^{2} d x d t+Y_{1}+Y_{2}+Y_{3} .
\end{aligned}
$$


ANALYSIS OF THE HEART-TORSO CONDUCTIVITY PARAMETERS RECOVERY INVERSE PROBLEM IN CARDIAC ELECTROPHYSIOLOGY ECG

Consequently, from (2.16), we can deduce the following inequality

$$
\begin{aligned}
\left\|L_{1}(z)\right\|_{2}^{2}+\left\|L_{2}(z)\right\|_{2}^{2}+2 s^{3} \lambda^{4} \int_{Q} \varphi^{3}|z|^{2} \sigma^{2}|\nabla \beta|^{4} d x d t+4 s \lambda^{2} \int_{Q} \varphi \sigma^{2}|\nabla z \cdot \nabla \beta|^{2} d x d t \\
+2 s \lambda^{2} \int_{Q} \varphi|\sigma \nabla \beta|^{2}|\nabla z|^{2} d x d t-4 s^{3} \lambda^{3} \int_{\Sigma_{\mathrm{ext}}} \varphi^{3}\left|\sigma_{\mathbf{t}} \partial_{n} \beta_{\mathbf{t}}\right|^{2} \partial_{n} \beta_{\mathbf{t}}\left|z_{\mathbf{t}}\right|^{2} d x d t \\
\quad-4 s^{2} \lambda^{3} \int_{\Sigma_{\mathrm{ext}}} \varphi^{2}\left|\sigma_{\mathbf{t}} \partial_{n} \beta_{\mathbf{t}}\right|^{2} \partial_{n} \beta_{\mathbf{t}}\left|z_{\mathbf{t}}\right|^{2} d x d t+2 s \lambda \int_{\Sigma} \varphi\left|\sigma \partial_{n} z\right|^{2}\left[\partial_{n} \beta\right]_{S} d x d t \\
-2 s \lambda \int_{\Sigma} \varphi\left(\sigma \partial_{n} \beta\right)\left|\nabla_{\tau} z\right|^{2}[\sigma]_{S} d x d t+2 s^{3} \lambda^{3} \int_{\Sigma} \varphi^{3}\left|\sigma \partial_{n} \beta\right|^{2}\left[\partial_{n} \beta\right]_{S}|z|^{2} d x d t \\
\leqslant\left\|F_{s}(z)\right\|_{2}^{2}+2\left(\left|Y_{1}\right|+\left|Y_{2}\right|+\left|Y_{3}\right|\right) .
\end{aligned}
$$

On the other hand, using the expression of $F_{s}(z)$, we get

$$
\left\|F_{s}(z)\right\|_{2}^{2} \leqslant\left\|e^{-s \eta} F\right\|_{2}^{2}+C s^{2} \lambda^{4} \int_{Q} \varphi^{3}|z|^{2} d x d t
$$

It is not difficult to deduce that the interface integrals are positive and (2.24), (2.26) and (2.28) yield that

$$
\begin{aligned}
\left|Y_{1}\right| \leqslant & C s \lambda \int_{Q} \varphi|\nabla z|^{2} d x d t+C s \lambda \int_{\Sigma_{\mathrm{ext}}} \varphi\left|\nabla_{\tau} z_{\mathbf{t}}\right|^{2} d x d t \\
\left|Y_{2}\right| \leqslant & \left(C_{\epsilon} s \lambda^{3} \int_{Q} \varphi^{3}|z|^{2} d x d t+\epsilon s \lambda^{2} \int_{Q} \varphi|\nabla z|^{2} d x d t\right. \\
& \left.+C_{\epsilon} s \lambda^{3} \int_{\Sigma} \varphi^{3}|z|^{2}\left|\sigma \partial_{n} \beta\right|^{2}\left[\partial_{n} \beta\right]_{S} d x d t+\epsilon s \lambda \int_{\Sigma} \varphi\left|\sigma \partial_{n} z\right|^{2}\left[\partial_{n} \beta\right]_{S} d x d t\right) \\
\left|Y_{3}\right| \leqslant & C s^{3} \lambda^{3} \int_{Q} \varphi^{3}|z|^{2} d x d t
\end{aligned}
$$

Thereafter, using the above expressions and taking $\epsilon$ small and $s$ large, by (2.30) we find

$$
\begin{aligned}
&\left\|L_{1, s}(z)\right\|_{2}^{2}+\left\|L_{2, s}(z)\right\|_{2}^{2}+2 s^{3} \lambda^{4} \int_{Q} \varphi^{3}|z|^{2} \sigma^{2}|\nabla \beta|^{4} d x d t+4 s \lambda^{2} \int_{Q} \varphi \sigma^{2}|\nabla z \cdot \nabla \beta|^{2} d x d t \\
&+2 s \lambda^{2} \int_{Q} \varphi|\sigma \nabla \beta|^{2}|\nabla z|^{2} d x d t-4 s^{3} \lambda^{3} \int_{\Sigma_{\mathrm{ext}}} \varphi^{3}\left|\sigma_{\mathbf{t}} \partial_{n} \beta_{\mathbf{t}}\right|^{2} \partial_{n} \beta_{\mathbf{t}}\left|z_{\mathbf{t}}\right|^{2} d x d t \\
&-4 s^{2} \lambda^{3} \int_{\Sigma_{\mathrm{ext}}} \varphi^{2}\left|\sigma_{\mathbf{t}} \partial_{n} \beta_{\mathbf{t}}\right|^{2} \partial_{n} \beta_{\mathbf{t}}\left|z_{\mathbf{t}}\right|^{2} d x d t+2 s \lambda \int_{\Sigma} \varphi\left|\sigma \partial_{n} z\right|^{2}\left[\partial_{n} \beta\right]_{S} d x d t \\
&-2 s \lambda \int_{\Sigma} \varphi\left(\sigma \partial_{n} \beta\right)\left|\nabla_{\tau} z\right|^{2}[\sigma]_{S} d x d t+2 s^{3} \lambda^{3} \int_{\Sigma} \varphi^{3}\left|\sigma \partial_{n} \beta\right|^{2}\left[\partial_{n} \beta\right]_{S}|z|^{2} d x d t \\
& \leqslant\left(\left\|e^{-s \eta} F\right\|_{2}^{2}+s^{2} \lambda^{4} \int_{Q} \varphi^{3}|z|^{2} d x d t+C s^{3} \lambda^{3} \int_{Q} \varphi^{3}|z|^{2} d x d t+\epsilon s \lambda^{2} \int_{Q} \varphi|\nabla z|^{2} d x d t\right. \\
& \quad+s \lambda^{4} \int_{Q} \varphi^{3}|z|^{2} d x d t+C_{\epsilon} s \lambda^{3} \int_{\Sigma} \varphi^{3}|z|^{2}\left|\sigma \partial_{n} \beta\right|^{2}\left[\partial_{n} \beta\right]_{S} d x d t \\
&\left.\quad+\epsilon s \lambda \int_{\Sigma} \varphi\left|\sigma \partial_{n} z\right|^{2}\left[\partial_{n} \beta\right]_{S} d x d t+C s \lambda \int_{\Sigma_{\mathrm{ext}}} \varphi\left|\nabla_{\tau} z_{\mathbf{t}}\right|^{2} d x d t\right) .
\end{aligned}
$$


Then, from (2.2) we get

$$
\begin{aligned}
& \left\|L_{1, s}(z)\right\|_{2}^{2}+\left\|L_{2, s}(z)\right\|_{2}^{2}+2 s^{3} \lambda^{4} \int_{Q} \varphi^{3}|z|^{2} \sigma^{2}|\nabla \beta|^{4} d x d t+2 s \lambda^{2} \int_{Q} \varphi|\sigma \nabla \beta|^{2}|\nabla z|^{2} d x d t \\
& -4 s^{3} \lambda^{3} \int_{\Sigma_{\mathrm{ext}}} \varphi^{3}\left|\sigma_{\mathbf{t}} \partial_{n} \beta_{\mathbf{t}}\right|^{2} \partial_{n} \beta_{\mathbf{t}}\left|z_{\mathbf{t}}\right|^{2} d x d t-4 s^{2} \lambda^{3} \int_{\Sigma_{\mathrm{ext}}} \varphi^{2}\left|\sigma_{\mathbf{t}} \partial_{n} \beta_{\mathbf{t}}\right|^{2} \partial_{n} \beta_{\mathbf{t}}\left|z_{\mathbf{t}}\right|^{2} d x d t \\
& \left.\leqslant\left\|e^{-s \eta} F\right\|_{2}^{2}+C\left(s^{3} \lambda^{4} \int_{Q} \varphi^{3}|z|^{2} d x d t+s \lambda^{2} \int_{Q} \varphi|\nabla z|^{2} d x d t+s \lambda \int_{\Sigma_{\text {ext }}} \varphi\left|\nabla_{\tau} z_{\mathbf{t}}\right|^{2} d x d t\right)\right) .
\end{aligned}
$$

Using the fact that $\sigma^{2}|\nabla \beta|^{2}>0$ in $\left(\overline{\Omega \backslash \omega_{0}}\right)$ and $\partial_{n} \beta<0$ on $\Gamma_{\text {ext }}$, we get

$$
\begin{aligned}
\left\|L_{1, s}(z)\right\|_{2}^{2}+ & \left\|L_{2, s}(z)\right\|_{2}^{2}+C s^{3} \lambda^{4} \int_{Q} \varphi^{3}|z|^{2} d x d t+C s \lambda^{2} \int_{Q} \varphi|\nabla z|^{2} d x d t \leqslant\left\|e^{-s \eta} F\right\|_{2}^{2} \\
& +C\left(s^{3} \lambda^{4} \int_{\omega_{0} \times(0, T)} \varphi^{3}|z|^{2} d x d t+s \lambda^{2} \int_{\omega_{0} \times(0, T)} \varphi|\nabla z|^{2} d x d t+s \lambda \int_{\Sigma_{\text {ext }}} \varphi\left|\nabla_{\tau} z_{\mathbf{t}}\right|^{2} d x d t\right) .
\end{aligned}
$$

We aim now back to our original variables and we obtain

$$
\begin{aligned}
& \int_{Q}\left(s^{3} \lambda^{4} \varphi^{3}|u|^{2}+s \lambda^{2} \varphi|\nabla u|^{2}\right) e^{-2 s \eta} d x d t \leqslant \int_{Q}|F|^{2} e^{-2 s \eta} d x d t \\
& \quad+C\left(\int_{\omega_{0} \times(0, T)}\left(s^{3} \lambda^{4} \varphi^{3}\left|u_{\mathbf{h}}\right|^{2}+s \lambda^{2} \varphi\left|\nabla u_{\mathbf{h}}\right|^{2}\right) e^{-2 s \eta} d x d t+s \lambda \int_{\Sigma_{\text {ext }}} \varphi\left|\nabla_{\tau} u_{\mathbf{t}}\right|^{2} e^{-2 s \eta} d x d t\right) .
\end{aligned}
$$

We should now eliminate $\left|\nabla u_{\mathbf{h}}\right|^{2}$ in $\omega_{0}$. We redefine the following function $\rho \in C_{0}^{\infty}(\omega)$ such that $\rho \equiv$ 1 in $\omega_{0}$ and $\rho \geqslant 0$. Thereafter, multiplying the following equation $\operatorname{div}(\sigma \nabla u)=F$ by $s \lambda^{2} \varphi \rho u e^{-2 s \eta}$ and integrating on $\omega \times(0, T)$, we obtain

$$
s \lambda^{2} \int_{\omega_{0} \times(0, T)} \varphi\left|\nabla u_{\mathbf{h}}\right|^{2} e^{-2 s \eta} d x d t \leqslant \int_{Q}|F|^{2} e^{-2 s \eta} d x d t+C s^{3} \lambda^{4} \int_{\omega \times(0, T)} \varphi^{3}\left|u_{\mathbf{h}}\right|^{2} e^{-2 s \eta} d x d t .
$$

Finally, we have

$$
\begin{aligned}
\int_{Q}\left(s^{3} \lambda^{4} \varphi^{3}|u|^{2}+s \lambda^{2} \varphi|\nabla u|^{2}\right) e^{-2 s \eta} d x d t \leqslant \int_{Q}|F|^{2} e^{-2 s \eta} d x d t & +C s^{3} \lambda^{4} \int_{\omega \times(0, T)} \varphi^{3}\left|u_{\mathbf{h}}\right|^{2} e^{-2 s \eta} d x d t \\
& +C s \lambda \int_{\Sigma_{\text {ext }}} \varphi\left|\nabla_{\tau} u_{\mathbf{t}}\right|^{2} e^{-2 s \eta} d x d t .
\end{aligned}
$$

This completes the proof of (2.10).

2.4. Global Carleman for the coupled heart-torso system. In this section, we will establish the global Carleman estimate for the coupled heart-torso system. We consider now the following system :

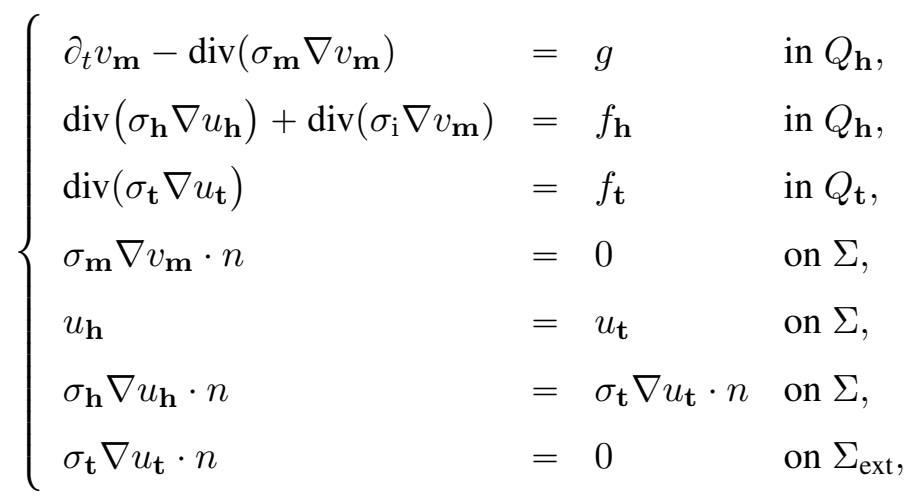

where $g \in L^{2}\left(Q_{\mathbf{h}}\right)$ and $f:=\left(f_{\mathbf{h}} \chi_{\mathbf{h}}+f_{\mathbf{t}} \chi_{\mathbf{t}}\right) \in L^{2}(Q)$. 
Theorem 2.3. Under the previous assumptions on $g$ and $f$, there exists $\lambda_{0}>0$ such that for any $\lambda \geqslant \lambda_{0}$ there exist $s_{0}:=s_{0}(\lambda)>0$ and $C=C_{\lambda}>0$ such that the solution $\left(v_{\mathbf{m}}, u\right) \in H^{1,2}\left(Q_{\mathbf{h}}\right) \times H^{1}(Q)$ to the system (2.37) satisfies

$$
\begin{aligned}
\int_{Q_{\mathbf{h}}}\left((s \varphi)\left(\left|\partial_{t} v_{\mathbf{m}}\right|^{2}+\left|\operatorname{div}\left(\sigma_{\mathbf{m}} \nabla v_{\mathbf{m}}\right)\right|^{2}\right)+(s \varphi)^{5}\left|v_{\mathbf{m}}\right|^{2}+(s \varphi)^{3}\left|\nabla v_{\mathbf{m}}\right|^{2}\right) e^{-2 s \eta} d x d t & \\
+\int_{Q}\left((s \varphi)^{3}|u|^{2}+(s \varphi)|\nabla u|^{2}\right) e^{-2 s \eta} d x d t & \\
\leqslant C\left(\int_{Q_{\mathbf{h}}}(s \varphi)^{2}|g|^{2} e^{-2 s \eta} d x d t+\right. & \int_{Q}|f|^{2} e^{-2 s \eta} d x d t+\int_{\Sigma_{e x t}}(s \varphi)\left|\nabla_{\tau} u_{\mathbf{t}}\right|^{2} e^{-2 s \eta} d x d t \\
& \left.+\int_{\omega \times(0, T)}\left((s \varphi)^{5}\left|v_{\mathbf{m}}\right|^{2}+(s \varphi)^{3}\left|u_{\mathbf{h}}\right|^{2}\right) e^{-2 s \eta} d x d t\right)
\end{aligned}
$$

for any $s>s_{0}$.

Proof. Applying Lemma 2.1 to $v_{\mathbf{m}}$ with $p=2$, we get

$$
\begin{aligned}
& \int_{Q_{\mathbf{h}}}\left((s \varphi)\left(\left|\partial_{t} v_{\mathbf{m}}\right|^{2}+\left|\operatorname{div}\left(\sigma_{\mathbf{m}} \nabla v_{\mathbf{m}}\right)\right|^{2}\right)+(s \varphi)^{5}\left|v_{\mathbf{m}}\right|^{2}+(s \varphi)^{3}\left|\nabla v_{\mathbf{m}}\right|^{2}\right) e^{-2 s \eta} d x d t \\
& \quad \leqslant C\left(\int_{Q_{\mathbf{h}}}(s \varphi)^{2}|g|^{2} e^{-2 s \eta} d x d t+\int_{\omega \times(0, T)}(s \varphi)^{5}\left|v_{\mathbf{m}}\right|^{2} e^{-2 s \eta} d x d t\right) .
\end{aligned}
$$

We apply now Theorem 2.2 to $u$ with $F_{\mathbf{h}}=f_{\mathbf{h}}-\operatorname{div}\left(\sigma_{\mathbf{i}} \nabla v_{\mathbf{m}}\right)$ and $F_{\mathbf{t}}=f_{\mathbf{t}}$, we obtain

$$
\begin{array}{r}
\int_{Q}\left((s \varphi)^{3}|u|^{2}+(s \varphi)|\nabla u|^{2}\right) e^{-2 s \eta} d x d t \leqslant C_{\lambda}\left(\int_{Q}|f|^{2} e^{-2 s \eta} d x d t+\int_{Q_{\mathbf{h}}}\left|\operatorname{div}\left(\sigma_{\mathbf{i}} \nabla v_{\mathbf{m}}\right)\right|^{2} e^{-2 s \eta} d x d t\right. \\
\left.+\int_{\omega \times(0, T)}(s \varphi)^{3} e^{-2 s \eta}\left|u_{\mathbf{h}}\right|^{2} d x d t+\int_{\Sigma_{\text {ext }}}(s \varphi)\left|\nabla_{\tau} u_{\mathbf{t}}\right|^{2} e^{-2 s \eta} d x d t\right) .
\end{array}
$$

Then, by summing (2.39) and (2.40) and taking $s$ large, we get

$$
\begin{aligned}
\int_{Q_{\mathbf{h}}}\left((s \varphi)\left(\left|\partial_{t} v_{\mathbf{m}}\right|^{2}+\left|\operatorname{div}\left(\sigma_{\mathbf{m}} \nabla v_{\mathbf{m}}\right)\right|^{2}\right)+(s \varphi)^{5}\left|v_{\mathbf{m}}\right|^{2}+(s \varphi)^{3}\left|\nabla v_{\mathbf{m}}\right|^{2}\right) e^{-2 s \eta} d x d t & \\
& +\int_{Q}\left((s \varphi)^{3}|u|^{2}+(s \varphi)|\nabla u|^{2}\right) e^{-2 s \eta} d x d t \\
\leqslant C\left(\int_{Q_{\mathbf{h}}}(s \varphi)^{2}|g|^{2} e^{-2 s \eta} d x d t+\right. & \int_{Q}|f|^{2} e^{-2 s \eta} d x d t+\int_{\Sigma_{\mathrm{ext}}}(s \varphi)\left|\nabla_{\tau} u_{\mathbf{t}}\right|^{2} e^{-2 s \eta} d x d t \\
& \left.+\int_{\omega \times(0, T)}\left((s \varphi)^{5}\left|v_{\mathbf{m}}\right|^{2}+(s \varphi)^{3}\left|u_{\mathbf{h}}\right|^{2}\right) e^{-2 s \eta} d x d t\right) .
\end{aligned}
$$

This completes the proof of (2.38).

\section{STABILITY OF THE CONDUCTIVITIES INVERSE PROBLEM}

This section devoted to proving Lipschitz stability given by Theorem 1.2 for our inverse problem which consists of identifying conductivities parameters $\sigma_{\mathbf{m}}$ and $\sigma_{\mathbf{t}}$ by means of Carleman estimate. We consider 
the following coupled heart torso system :

$$
\left\{\begin{array}{lll}
\partial_{t} v_{\mathbf{m}}-\operatorname{div}\left(\sigma_{\mathbf{m}} \nabla v_{\mathbf{m}}\right) & =I_{\text {app }}+I_{i o n}\left(\bar{\varrho}, v_{\mathbf{m}}, \mathbf{w}, \mathbf{z}\right) & \text { in } Q_{\mathbf{h}}, \\
\operatorname{div}\left(\sigma_{\mathbf{h}} \nabla u_{\mathbf{h}}\right) & =-\operatorname{div}\left(\sigma_{\mathrm{i}} \nabla v_{\mathbf{m}}\right) & \\
\operatorname{div}\left(\sigma_{\mathbf{t}} \nabla u_{\mathbf{t}}\right) & =0 & \text { in } Q_{\mathbf{h}}, \\
\partial_{t} \mathbf{w}-\mathbf{F}\left(v_{\mathbf{m}}, \mathbf{w}\right) & =0 & \text { in } Q_{\mathbf{t}}, \\
\partial_{t} \mathbf{z}-\mathbf{G}\left(\bar{\varrho}_{\mathbf{h}}, v_{\mathbf{m}}, \mathbf{w}, \mathbf{z}\right) & =0 & \text { in } Q_{\mathbf{h}},
\end{array}\right.
$$

with the following interface boundary conditions on $\Sigma$

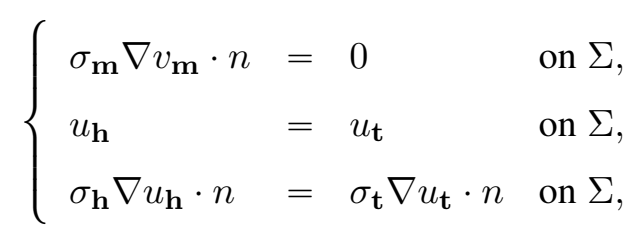

and the following interface boundary conditions on $\Sigma_{\text {ext }}$

$$
\sigma_{\mathbf{t}} \nabla u_{\mathbf{t}} \cdot n=0 \quad \text { in } \Sigma_{\text {ext }} .
$$

In the sequel and without loss of generality, we may assume that $t_{0}=T / 2$ and to simplify the notations, we denote $\eta_{0}(x)=\eta\left(x, t_{0}\right)$.

3.1. Preliminaries. We will prove at first a Carleman estimate for a first order partial differential operator which we will use to prove our stability result. Thereby, we consider the following first order partial differential operator $A(x, D)$ in a bounded domain $\Omega_{\mathbf{i}} \subset \mathbb{R}^{n}$

$$
A(x, D) y=\sum_{j=1}^{n} \gamma_{j}(x) \partial_{j} y+\gamma_{0}(x) y, \quad x \in \Omega_{\mathbf{i}},
$$

where

$$
\gamma_{0}(x) \in C\left(\bar{\Omega}_{\mathbf{i}}\right), \gamma=\left(\gamma_{1}, \ldots, \gamma_{n}\right) \in C^{1}(\bar{\Omega})^{n},\left\|\gamma_{j}\right\|_{C^{1}\left(\bar{\Omega}_{\mathbf{i}}\right)} \leqslant M, j=1, \ldots, n \text {, and }\left\|\gamma_{0}\right\|_{C^{1}\left(\bar{\Omega}_{\mathbf{i}}\right)} \leqslant M .
$$

Let $\tilde{\omega} \subset \Omega_{\mathbf{i}}$ (possibly empty) such that

$$
|\nabla \beta(x) \cdot \gamma(x)| \geqslant c_{0}, \quad \text { on } \bar{\Omega}_{\mathbf{i}} \mid \tilde{\omega} .
$$

Then, inspired by Lemma 2.1 in [7], we have

Lemma 3.1. There exist constants $s_{0}>0$ and $C>0$ such that the following estimate holds true

$$
s \int_{\Omega_{\mathbf{i}}}|y(x)|^{2} e^{-2 s \eta_{0}(x)} d x \leqslant C \int_{\Omega_{\mathbf{i}}}|A(x, D) y(x)|^{2} e^{-2 s \eta_{0}(x)} d x+s \int_{\tilde{\omega}}|y(x)|^{2} e^{-2 s \eta_{0}(x)} d x,
$$

for any $y \in H_{0}^{1}\left(\Omega_{\mathbf{i}}\right)$ and all $s>s_{0}$.

Proof. Let $\theta(x)=|\nabla \beta(x) \cdot \gamma(x)|$. We multiply the equation (3.4) by $\theta y e^{-2 s \eta_{0}(x)}$, we have

$$
\begin{aligned}
\int_{\Omega_{\mathrm{i}}} A y(x) \theta(x) y(x) e^{-2 s \eta_{0}(x)} d x=\int_{\Omega_{\mathbf{i}}} \nabla y(x) \cdot\left(e^{-2 s \eta_{0}(x)} \theta(x) y(x) \gamma(x)\right) d x & \\
& +\int_{\Omega_{\mathbf{i}}} \theta(x) \gamma_{0}(x)|y(x)|^{2} e^{-2 s \eta_{0}(x)} d x .
\end{aligned}
$$


Then, applying the divergence Theorem by taking into account that $y \in H_{0}^{1}(\Omega)$, we obtain

$$
\begin{aligned}
\int_{\Omega_{\mathrm{i}}} A y(x) \theta(x) y(x) e^{-2 s \eta_{0}(x)} d x=-\int_{\Omega_{\mathrm{i}}} y(x) \operatorname{div}( & \left.e^{-2 s \eta_{0}(x)} \theta(x) y(x) \gamma(x)\right) d x \\
& +\int_{\Omega_{\mathbf{i}}} \theta(x) \gamma_{0}(x)|y(x)|^{2} e^{-2 s \eta_{0}(x)} d x .
\end{aligned}
$$

Thereafter, we have

$$
\begin{gathered}
\int_{\Omega_{\mathbf{i}}} A y(x) \theta(x) y(x) e^{-2 s \eta_{0}(x)} d x=-2 s \int_{\Omega_{\mathbf{i}}} \varphi\left(x, t_{0}\right)|\theta(x)|^{2}|y(x)|^{2} e^{-2 s \eta_{0}(x)} d x \\
-\int_{\Omega_{\mathbf{i}}}|y(x)|^{2} \operatorname{div}(\theta(x) \gamma(x)) e^{-2 s \eta_{0}(x)} d x-\int_{\Omega_{\mathbf{i}}} y(x) \nabla y(x) \cdot \theta(x) \gamma(x) e^{-2 s \eta_{0}(x)} d x \\
\quad+\int_{\Omega_{\mathbf{i}}} \theta(x) \gamma_{0}(x)|y(x)|^{2} e^{-2 s \eta_{0}(x)} d x .
\end{gathered}
$$

Using the fact that

$$
\nabla y(x) \cdot \gamma(x)=A(x, D) y(x)-\gamma_{0} y(x)
$$

we get

$$
\begin{aligned}
& 2 s \int_{\Omega_{\mathbf{i}}}|y(x)|^{2} \varphi\left(x, t_{0}\right)|\theta(x)|^{2} e^{-2 s \eta_{0}(x)} d x=2 \int_{\Omega_{\mathbf{i}}} \theta(x) \gamma_{0}(x)|y(x)|^{2} e^{-2 s \eta_{0}(x)} d x \\
& \quad-2 \int_{\Omega_{\mathrm{i}}} A(x, D) y(x) \cdot \theta(x) y(x) e^{-2 s \eta_{0}(x)} d x-\int_{\Omega_{\mathbf{i}}}|y(x)|^{2} \operatorname{div}(\theta(x) \gamma(x)) e^{-2 s \eta_{0}(x)} d x .
\end{aligned}
$$

Thereafter, applying Cauchy-Schwartz inequality to (3.9) and from (3.5) we obtain

$$
\left.2 s \int_{\Omega_{\mathbf{i}}} \varphi\left(x, t_{0}\right)|y(x)|^{2}|| \theta(x)\right|^{2} e^{-2 s \eta_{0}(x)} d x \leqslant C \int_{\Omega_{\mathbf{i}}}\left(|A(x, D) y(x)|^{2}+|y(x)|^{2}\right) e^{-2 s \eta_{0}(x)} d x .
$$

In order to minorate the left hand side quantity, we use the condition (3.6) and it is easy to deduce the desired estimate (3.7). This completes the proof.

So as to estimate the gating variable $\mathbf{w}$ and the ionic concentration $\mathbf{z}$, we need to state the following Lemma.

Lemma 3.2. There exists $C>0$ such that the following estimate

$$
\begin{aligned}
\int_{Q} e^{-2 s \eta(x, t)} \ell(t)^{-2}|u(x, t)|^{2} d x d t \leqslant C\left(\int_{Q} e^{-2 s \eta(x, t)} \ell(t)^{-2}\left|u\left(x, t_{0}\right)\right|^{2} d x d t\right. & \\
& \left.+s^{-1} \int_{Q} e^{-2 s \eta(x, t)}\left|\partial_{t} u(x, t)\right|^{2} d x d t\right),
\end{aligned}
$$

holds for any $u \in H^{1}\left(0, T, L^{2}(\Omega)\right)$ and any $s>0$.

Proof. By applying the Cauchy-Schwartz inequality, we get

$$
\int_{Q}\left|\int_{t_{0}}^{t} \partial_{t} u(x, \tau) d \tau\right|^{2} \ell(t)^{-2} e^{-2 s \eta(x, t)} d x d t \leqslant \int_{Q}\left(\int_{t_{0}}^{t}\left|\partial_{t} u(x, \tau)\right|^{2} d \tau\right)\left(t-t_{0}\right) \ell(t)^{-2} e^{-2 s \eta(x, t)} d x d t .
$$

Then, using the fact that

$$
\partial_{t} \eta(x, t)=\frac{2\left(t-t_{0}\right)}{\ell(t)^{2}}\left(e^{2 \lambda\|\beta\|_{\infty}}-e^{\lambda \beta}\right)=2\left(t-t_{0}\right) \ell(t)^{-2} \tilde{h}(x),
$$

where $\tilde{h}(x)=\left(e^{2 \lambda\|\beta\|_{\infty}}-e^{\lambda \beta}\right)$. Moreover, we have

$$
\partial_{t} \eta(x, t) \leqslant 0, \quad \int_{t_{0}}^{t}\left|\partial_{t} u(x, \tau)\right|^{2} d \tau \leqslant 0, \quad \text { for } \quad 0 \leqslant t \leqslant t_{0},
$$


and

$$
\partial_{t} \eta(x, t) \geqslant 0, \quad \int_{t_{0}}^{t}\left|\partial_{t} u(x, \tau)\right|^{2} d \tau \geqslant 0, \quad \text { for } \quad t_{0} \leqslant t \leqslant T .
$$

Thereafter, we can deduce that

$$
\begin{aligned}
\int_{Q}\left|\int_{t_{0}}^{t} \partial_{t} u(x, \tau) d \tau\right|^{2} \ell(t)^{-2} e^{-2 s \eta(x, t)} d x d t & \leqslant C \int_{Q}\left(\int_{t_{0}}^{t}\left|\partial_{t} u(x, \tau)\right|^{2} d \tau\right) \partial_{t} \eta(x, t) e^{-2 s \eta(x, t)} d x d t \\
& =-\frac{C}{2 s} \int_{Q}\left(\int_{t_{0}}^{t}\left|\partial_{t} u(x, \tau)\right|^{2} d \tau\right) \partial_{t}\left(e^{-2 s \eta(x, t)}\right) d x d t
\end{aligned}
$$

Subsequently, integration by parts with respect to time variable and taking into account that $e^{-2 s \eta(x, T)}=$ $e^{-2 s \eta(x, 0)}=0$, we get

$$
-\frac{C}{2 s} \int_{Q}\left(\int_{t_{0}}^{t}\left|\partial_{t} u(x, \tau)\right|^{2} d \tau\right) \partial_{t}\left(e^{-2 s \eta(x, t)}\right) d x d t=\frac{C}{2 s} \int_{Q}\left|\partial_{t} u(x, t)\right|^{2} e^{-2 s \eta(x, t)} d x d t .
$$

On the other hand, we have

$$
\int_{Q}\left|\int_{t_{0}}^{t} \partial_{t} u(x, \tau) d \tau\right|^{2} \ell(t)^{-2} e^{-2 s \eta(x, t)} d x d t=\int_{Q}\left|u(x, t)-u\left(x, t_{0}\right)\right|^{2} \ell(t)^{-2} e^{-2 s \eta(x, t)} d x d t .
$$

Since

$$
\ell(t)^{-2}|u(x, t)|^{2} \leqslant C\left(\ell(t)^{-2}\left|u\left(x, t_{0}\right)\right|^{2}+\ell(t)^{-2}\left|u(x, t)-u\left(x, t_{0}\right)\right|^{2}\right),
$$

and from (3.12), (3.13) and (3.14), we can deduce (3.11). This complete the proof.

Finally, we have the following Lemma ( See [24]).

Lemma 3.3. Let $m \geqslant 1$ and $\Omega$ a bounded domain of $\mathbb{R}^{n}$. Then, there exist $C>0, s_{0}>0$ such that the following estimate holds

$$
\int_{Q} \ell(t)^{-m} e^{-2 s \eta(x, t)}|k(x)|^{2} d x d t \leqslant \frac{C}{\sqrt{s}} \int_{\Omega} e^{-2 s \eta_{0}(x)}|k(x)|^{2} d x,
$$

for all $k \in L^{2}(\Omega)$ and any $s \geqslant s_{0}$.

3.2. Linearized inverse problem. Let consider two sets of admissible coefficients $\left(\sigma_{\mathbf{m}}, \sigma_{\mathbf{h}}, \sigma_{\mathbf{i}}, \sigma_{\mathbf{t}}\right)$ and $\left(\widehat{\sigma}_{\mathbf{m}}, \widehat{\sigma}_{\mathbf{h}}, \hat{\sigma}_{\mathbf{i}}, \widehat{\sigma}_{\mathbf{t}}\right)$ and the corresponding solutions $\left(u_{\mathbf{h}}, u_{\mathbf{t}}, v_{\mathbf{m}}, \mathbf{w}, \mathbf{z}\right)$ and $\left(\widehat{u}_{\mathbf{h}}, \widehat{u}_{\mathbf{t}}, \widehat{v}_{\mathbf{m}}, \widehat{\mathbf{w}}, \widehat{\mathbf{z}}\right)$. Then, we define the difference as follows:

$$
\begin{aligned}
& \tilde{v}_{\mathbf{m}}=v_{\mathbf{m}}-\widehat{v}_{\mathbf{m}}, \quad \tilde{u}_{\mathbf{h}}=u_{\mathbf{h}}-\widehat{u}_{\mathbf{h}}, \quad \tilde{u}_{\mathbf{t}}=u_{\mathbf{t}}-\widehat{u}_{\mathbf{t}}, \quad \tilde{\mathbf{w}}=\mathbf{w}-\widehat{\mathbf{w}}, \quad \tilde{\mathbf{z}}=\mathbf{z}-\widehat{\mathbf{z}}, \\
& \text { and } \quad \tilde{\sigma}_{\mathbf{j}}=\sigma_{\mathbf{j}}-\widehat{\sigma}_{\mathbf{j}}, \text { with } \mathbf{j} \in\{\mathbf{m}, \mathbf{i}, \mathbf{e}, \mathbf{h}, \mathbf{t}\} .
\end{aligned}
$$

Thereafter, we easily see that $\left(\tilde{u}_{\mathbf{h}}, \tilde{u}_{\mathbf{t}}, \tilde{v}_{\mathbf{m}}, \tilde{\mathbf{w}}, \tilde{\mathbf{z}}\right)$ satisfies the following system

$$
\left\{\begin{array}{lll}
\partial_{t} \tilde{v}_{\mathbf{m}}-\operatorname{div}\left(\sigma_{\mathbf{m}} \nabla \tilde{v}_{\mathbf{m}}\right) & =\operatorname{div}\left(\tilde{\sigma}_{\mathbf{m}} \nabla \widehat{v}_{\mathbf{m}}\right)+R(x, t) & \text { in } Q_{\mathbf{h}}, \\
\operatorname{div}\left(\sigma_{\mathbf{h}} \nabla \tilde{u}_{\mathbf{h}}\right)+\operatorname{div}\left(\sigma_{\mathrm{i}} \nabla \tilde{v}_{\mathbf{m}}\right) & =-\operatorname{div}\left(\tilde{\sigma}_{\mathrm{i}} \nabla \widehat{v}_{\mathbf{m}}\right)-\operatorname{div}\left(\tilde{\sigma}_{\mathbf{h}} \nabla \widehat{u}_{\mathbf{h}}\right) & \text { in } Q_{\mathbf{h}}, \\
\operatorname{div}\left(\sigma_{\mathbf{t}} \nabla \tilde{u}_{\mathbf{t}}\right) & =-\operatorname{div}\left(\tilde{\sigma}_{\mathbf{t}} \nabla \widehat{u}_{\mathbf{t}}\right) & \text { in } Q_{\mathbf{t}}, \\
\partial_{t} \tilde{\mathbf{w}}-\mathbf{L}\left(v_{\mathbf{m}}, \mathbf{w}, \widehat{v}_{\mathbf{m}}, \widehat{\mathbf{w}}\right) & =0 & \text { in } Q_{\mathbf{h}}, \\
\partial_{t} \tilde{\mathbf{z}}-\mathbf{K}\left(\bar{\varrho}, v_{\mathbf{m}}, \mathbf{w}, \mathbf{z}, \widehat{v}_{\mathbf{m}}, \widehat{\mathbf{w}}, \widehat{\mathbf{z}}\right) & =0 & \text { in } Q_{\mathbf{h}},
\end{array}\right.
$$


ANALYSIS OF THE HEART-TORSO CONDUCTIVITY PARAMETERS RECOVERY INVERSE PROBLEM IN CARDIAC ELECTROPHYSIOLOGY ECG with the following interface boundary conditions on $\Sigma$

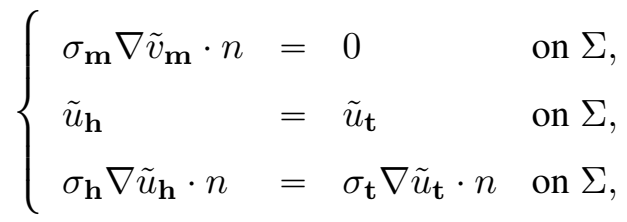

and the following interface boundary conditions on $\Sigma_{\text {ext }}$

$$
\sigma_{\mathbf{t}} \nabla \tilde{u}_{\mathbf{t}} \cdot n=0 \quad \Sigma_{\text {ext }} \cdot
$$

where we have assumed that $\sigma_{j}=\hat{\sigma}_{j}$ on $S$ and we denote $\tilde{\mathbf{F}}, \tilde{\mathbf{G}}$ and $R(x, t)$ as follows :

$$
\begin{gathered}
\mathbf{L}\left(v_{\mathbf{m}}, \mathbf{w}, \widehat{v}, \widehat{\mathbf{w}}\right)=\mathbf{F}\left(v_{\mathbf{m}}, \mathbf{w}\right)-\mathbf{F}(\widehat{v}, \widehat{\mathbf{w}}), \\
\mathbf{K}\left(\bar{\varrho}, v_{\mathbf{m}}, \mathbf{w}, \mathbf{z}, \widehat{v}_{\mathbf{m}}, \widehat{\mathbf{w}}, \widehat{\mathbf{z}}\right)=\mathbf{G}\left(\bar{\varrho}, v_{\mathbf{m}}, \mathbf{w}, \mathbf{z}\right)-\mathbf{G}\left(\bar{\varrho}, \widehat{v}_{\mathbf{m}}, \widehat{\mathbf{w}}, \widehat{\mathbf{z}}\right),
\end{gathered}
$$

and

$$
R(x, t)=I_{i o n}\left(\bar{\varrho}, v_{\mathbf{m}}, \mathbf{w}, \mathbf{z}\right)-I_{\text {ion }}\left(\bar{\varrho}, \widehat{v}_{\mathbf{m}}, \widehat{\mathbf{w}}, \widehat{\mathbf{z}}\right) .
$$

where $R(x, t)$ satisfies the following estimates (see Lemma 5.1 in [1]) :

$$
|R(x, t)|^{2} \leqslant C\left(\left|\tilde{v}_{\mathbf{m}}(x, t)\right|^{2}+|\tilde{\mathbf{w}}(x, t)|^{2}+|\tilde{\mathbf{z}}(x, t)|^{2}\right)
$$

and

$$
\left|\partial_{t} R(x, t)\right|^{2} \leqslant C\left(\left|\tilde{v}_{\mathbf{m}}(x, t)\right|^{2}+\left|\partial_{t} \tilde{v}_{\mathbf{m}}(x, t)\right|^{2}+|\tilde{\mathbf{w}}(x, t)|^{2}\right) .
$$

Lemma 3.4. Let $\left(\tilde{u}_{\mathbf{h}}, \tilde{u}_{\mathbf{t}}, \tilde{v}_{\mathbf{m}}, \tilde{\boldsymbol{w}}, \tilde{\boldsymbol{z}}\right)$ solution of the linearized problem (3.16). Then, there exists a constant $C>0$ such that we have the following estimate:

$$
\left|\partial_{t}^{2} R(x, t)\right|^{2} \leqslant C\left(\sum_{j=0}^{2}\left|\partial_{t}^{j} \tilde{v}_{\mathbf{m}}(x, t)\right|^{2}+|\tilde{\boldsymbol{w}}(x, t)|^{2}+|\tilde{z}(x, t)|^{2}\right) .
$$

Proof. We proceed as Lemma 5.1 in [1], we get

$$
\left|\partial_{t}^{2} R(x, t)\right|^{2} \leqslant C\left(\sum_{j=0}^{2}\left|\partial_{t}^{j} \tilde{v}_{\mathbf{m}}(x, t)\right|^{2}+|\tilde{\mathbf{w}}(x, t)|^{2}+|\tilde{\mathbf{z}}(x, t)|^{2}+\left|\partial_{t}^{2} \tilde{\mathbf{w}}(x, t)\right|^{2}+\left|\partial_{t}^{2} \tilde{\mathbf{z}}(x, t)\right|^{2}\right) .
$$

Thereafter, we recall that $\tilde{\mathbf{w}}:=\left(\tilde{w}_{1}, \ldots, \tilde{w}_{k}\right)$ and $\partial_{t} \tilde{\mathbf{w}}=\mathbf{F}\left(v_{\mathbf{m}}, \mathbf{w}\right)-\mathbf{F}\left(\widehat{v}_{\mathbf{m}}, \widehat{\mathbf{w}}\right)$ and by a derivative with respect to $t$, we get

$$
\partial_{t}^{2} \tilde{w}_{j}=\partial_{t}\left(F_{j}\left(v_{\mathbf{m}}, w_{j}\right)-F_{j}\left(\widehat{v}_{\mathbf{m}}, \widehat{w}_{j}\right)\right), \quad \forall j=1, \ldots, k
$$

Then, we have

$$
\begin{aligned}
\partial_{t}^{2} \tilde{w}_{j}=\partial_{t} \tilde{v}_{\mathbf{m}} \partial_{1} F_{j}\left(v_{\mathbf{m}}, w_{j}\right) & +\partial_{t} \tilde{w}_{j} \partial_{2} F_{j}\left(v_{\mathbf{m}}, w_{j}\right)+\partial_{t} \widehat{v}_{\mathbf{m}}\left(\partial_{1} F_{j}\left(v_{\mathbf{m}}, w_{j}\right)-\partial_{1} F_{j}\left(\widehat{v}_{\mathbf{m}}, \widehat{w}_{j}\right)\right) \\
& +\partial_{t} \widehat{w}_{j}\left(\partial_{2} F_{j}\left(v_{\mathbf{m}}, w_{j}\right)-\partial_{2} F_{j}\left(\widehat{v}_{\mathbf{m}}, \widehat{w}_{j}\right)\right)
\end{aligned}
$$

where $\partial_{l}$ represent the partial derivative with respect to the $l^{\text {th }}$ variable $l=1,2$. Using the fact that $F_{j}$ is locally Lipschitz and the a priori boundedness of the solutions (A.1), we get

$$
\begin{aligned}
\left|\partial_{t}^{2} \tilde{\mathbf{w}}\right|^{2} & \leqslant C\left(\left|\tilde{v}_{\mathbf{m}}\right|^{2}+\left|\partial_{t} \tilde{v}_{\mathbf{m}}\right|^{2}+|\tilde{\mathbf{w}}|^{2}+\left|\partial_{t} \tilde{\mathbf{w}}\right|^{2}\right) \\
& \leqslant C\left(\left|\tilde{v}_{\mathbf{m}}\right|^{2}+\left|\partial_{t} \tilde{v}_{\mathbf{m}}\right|^{2}+|\tilde{\mathbf{w}}|^{2}\right) .
\end{aligned}
$$

Similarly, we have $\tilde{\mathbf{z}}:=\left(\tilde{z}_{1}, \ldots, \tilde{z}_{\mathbf{m}}\right)$ and $\partial_{t} \tilde{\mathbf{z}}=\mathbf{G}\left(\bar{\varrho}, v_{\mathbf{m}}, \mathbf{w}, \mathbf{z}\right)-\mathbf{G}\left(\bar{\varrho}, \widehat{v}_{\mathbf{m}}, \widehat{\mathbf{w}}, \widehat{\mathbf{z}}\right)$ with $G$ is defined as follows

$$
G_{\mathbf{i}}\left(\bar{\varrho}, v_{\mathbf{m}}, \mathbf{w}, \mathbf{z}\right):=-J_{\mathbf{i}}\left(\bar{\varrho}, v_{\mathbf{m}}, \mathbf{w}, \log z_{\mathbf{i}}\right)+H_{\mathbf{i}}\left(\bar{\varrho}, v_{\mathbf{m}}, \mathbf{w}, \mathbf{z}\right), \quad \forall i=1, \ldots, m .
$$


By a derivative with respect to $t$, we get

$$
\begin{aligned}
\partial_{t}^{2} \tilde{z}_{\mathbf{i}} & =\partial_{t}\left(G_{\mathbf{i}}(\bar{\varrho}, v, \mathbf{w}, \mathbf{z})-G_{\mathbf{i}}\left(\bar{\varrho}, \widehat{v}_{\mathbf{m}}, \widehat{\mathbf{w}}, \widehat{\mathbf{z}}\right)\right) \\
& =\partial_{t}\left(-J_{\mathbf{i}}\left(\bar{\varrho}, v_{\mathbf{m}}, \mathbf{w}, \log z_{\mathbf{i}}+H_{\mathbf{i}}\left(\bar{\varrho}, v_{\mathbf{m}}, \mathbf{w}, \mathbf{z}\right)\right)+\partial_{t}\left(J_{\mathbf{i}}\left(\bar{\varrho}, \widehat{v}_{\mathbf{m}}, \widehat{\mathbf{w}}, \log \widehat{z}_{\mathbf{i}}\right)-H_{\mathbf{i}}\left(\bar{\varrho}, \widehat{v}_{\mathbf{m}}, \widehat{\mathbf{w}}, \widehat{\mathbf{z}}\right)\right)(3.28)\right.
\end{aligned}
$$

where

$$
\partial_{t}\left(J_{\mathbf{i}}\left(\bar{\varrho}, v_{\mathbf{m}}, \mathbf{w}, \log z_{\mathbf{i}}\right)\right)=\partial_{t} v_{\mathbf{m}} \partial_{2} J_{\mathbf{i}}+\sum_{j=1}^{k} \partial_{t} w_{j} \partial_{j+2} J_{\mathbf{i}}+\partial_{t} \log z_{\mathbf{i}} \partial_{k+3} J_{\mathbf{i}}
$$

and

$$
\partial_{t}\left(H_{\mathbf{i}}\left(\bar{\varrho}, v_{\mathbf{m}}, \mathbf{w}, \mathbf{z}\right)\right)=\partial_{t} v_{\mathbf{m}} \partial_{2} H_{\mathbf{i}}+\sum_{j=1}^{k} \partial_{t} w_{j} \partial_{j+2} H_{\mathbf{i}}+\sum_{j=1}^{m} \partial_{t} z_{j} \partial_{k+2+j} H_{\mathbf{i}}
$$

Thus, by (3.28), (3.29), (3.30) and the a priori boundedness of the solutions, we have

$$
\begin{aligned}
\left|\partial_{t}^{2} \tilde{\mathbf{z}}\right|^{2} & \leqslant C\left(\left|\tilde{v}_{\mathbf{m}}\right|^{2}+\left|\partial_{t} \tilde{v}_{\mathbf{m}}\right|^{2}+|\tilde{\mathbf{w}}|^{2}+|\tilde{\mathbf{z}}|^{2}+\left|\partial_{t} \tilde{\mathbf{w}}\right|^{2}+\left|\partial_{t} \tilde{\mathbf{z}}\right|^{2}\right) \\
& \leqslant C\left(\left|\tilde{v}_{\mathbf{m}}\right|^{2}+\left|\partial_{t} \tilde{v}_{\mathbf{m}}\right|^{2}+|\tilde{\mathbf{w}}|^{2}+|\tilde{\mathbf{z}}|^{2}\right)
\end{aligned}
$$

since $J_{\mathbf{i}}$ and $H_{\mathbf{i}}$ are locally Lipschitz. Subsequently, from (3.25),(3.27), (3.31) we obtain (3.24).

In the rest of this section, let

$$
\tilde{v}_{\mathbf{m}}^{(j)}=\partial_{t}^{j} \tilde{v}_{\mathbf{m}}, \quad \tilde{u}^{(j)}=\partial_{t}^{j} \tilde{u}, \quad \widehat{v}^{(j)}=\partial_{t}^{j} \widehat{v} \quad \text { and } \quad \widehat{u}^{(j)}=\partial_{t}^{j} \widehat{u}, \quad j=0,1,2 .
$$

Now, by considering the time derivative of the system (3.16)-(3.17)-(3.18), we obtain the following system

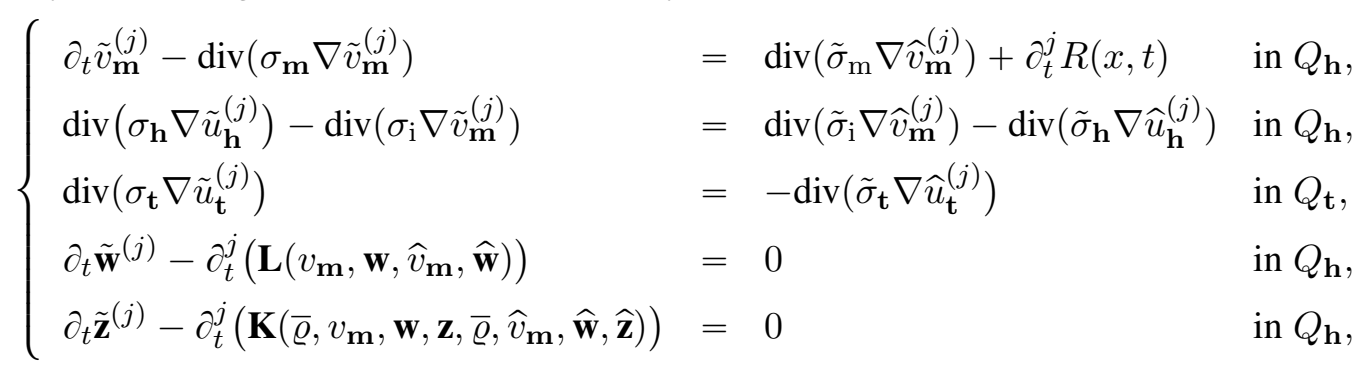

with the following interface boundary conditions on $\Sigma$

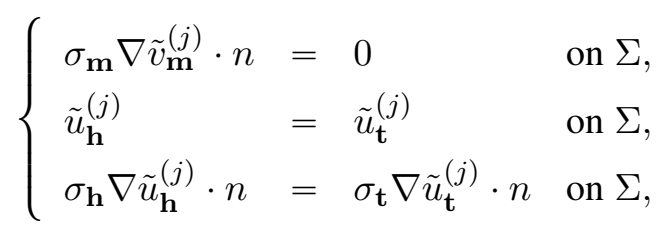

and the following interface boundary conditions on $\Sigma_{\text {ext }}$

In the next, we use the following notations

$$
\sigma_{\mathbf{t}} \nabla \tilde{u}_{\mathbf{t}}^{(j)} \cdot n=0 \quad \text { on } \Sigma_{\text {ext }} .
$$

$$
\begin{aligned}
M_{\omega}^{2}\left(\tilde{v}_{\mathbf{m}}, \tilde{u}_{\mathbf{h}}\right) & =\left\|\tilde{v}_{\mathbf{m}}\right\|_{H^{2}\left(0, T ; L^{2}(\omega)\right)}^{2}+\left\|\tilde{u}_{\mathbf{h}}\right\|_{H^{2}\left(0, T ; L^{2}(\omega)\right)}^{2}, \\
M_{\Gamma_{\mathrm{ext}}}^{2}\left(\tilde{u}_{\mathbf{t}}\right) & =\left\|\tilde{u}_{\mathbf{t}}\right\|_{H^{2}\left(0, T ; H^{1}\left(\Gamma_{\mathrm{ext}}\right)\right)}^{2}, \\
M_{t_{0}}^{2}\left(\tilde{v}_{\mathbf{m}}, \tilde{u}_{\mathbf{t}}, \tilde{\mathbf{w}}, \tilde{\mathbf{z}}\right) & =\left\|\tilde{v}_{\mathbf{m}}\left(\cdot, t_{0}\right)\right\|_{H^{2}\left(\Omega_{\mathbf{h}}\right)}^{2}+\left\|\tilde{u}_{\mathbf{t}}\left(\cdot, t_{0}\right)\right\|_{H^{2}\left(\Omega_{\mathbf{t}}\right)}^{2} \\
& \quad+\left\|\tilde{\mathbf{w}}\left(\cdot, t_{0}\right)\right\|_{H^{1}\left(\Omega_{\mathbf{h}}\right)}^{2}+\left\|\tilde{\mathbf{z}}\left(\cdot, t_{0}\right)\right\|_{H^{1}\left(\Omega_{\mathbf{h}}\right)}^{2} .
\end{aligned}
$$

We recall the assumption (A.2) for $\left(\widehat{v}_{\mathbf{m}}, \widehat{u}_{\mathbf{h}}, \widehat{u}_{\mathbf{t}}\right)$

$$
\left\|\widehat{v}_{\mathbf{m}}\right\|_{W^{2, \infty}\left(0, T ; W^{2, \infty}\left(\Omega_{\mathbf{h}}\right)\right)}+\left\|\widehat{u}_{\mathbf{h}}\right\|_{W^{2, \infty}\left(0, T ; W^{2, \infty}\left(\Omega_{\mathbf{h}}\right)\right)}+\left\|\widehat{u}_{\mathbf{t}}\right\|_{W^{2, \infty}\left(0, T ; W^{2, \infty}\left(\Omega_{\mathbf{t}}\right)\right)} \leqslant M
$$

for some positive constant $M$. 
ANALYSIS OF THE HEART-TORSO CONDUCTIVITY PARAMETERS RECOVERY INVERSE PROBLEM IN CARDIAC ELECTROPHYSIOLOGY ECG

Lemma 3.5. Let $\left(\tilde{v}_{\mathbf{m}}, \tilde{u}_{\mathbf{h}}, \tilde{u}_{\mathbf{t}}, \tilde{\boldsymbol{w}}, \tilde{\boldsymbol{z}}\right)$ solution of the linearized problem (3.16)-(3.17)-(3.18). Then, there exist constants $s_{0}>0$ and $C>0$ such that the following estimate holds

$$
\begin{aligned}
& \sum_{j=0}^{2}\left(\int_{Q_{\mathbf{h}}}\left(\left(\frac{s}{\ell(t)}\right)\left(\left|\partial_{t} \tilde{v}_{\mathbf{m}}^{(j)}\right|^{2}+\left|\operatorname{div}\left(\sigma_{\mathbf{m}} \nabla \tilde{v}_{\mathbf{m}}^{(j)}\right)\right|^{2}\right)+\left(\frac{s}{\ell(t)}\right)^{5}\left|\tilde{v}_{\mathbf{m}}^{(j)}\right|^{2}+\left(\frac{s}{\ell(t)}\right)^{3}\left|\nabla \tilde{v}_{\mathbf{m}}^{(j)}\right|^{2}\right) e^{-2 s \eta} d x d t\right. \\
& \left.+\int_{Q}\left(\left(\frac{s}{\ell(t)}\right)^{3}\left|\tilde{u}^{(j)}\right|^{2}+\left(\frac{s}{\ell(t)}\right)\left|\nabla \tilde{u}^{(j)}\right|^{2}\right) e^{-2 s \eta} d x d t\right) \leqslant C\left(\int_{Q_{\mathbf{h}}}\left(\frac{s}{\ell(t)}\right)^{2}\left(\left|\tilde{\sigma}_{\mathbf{i}}\right|^{2}+\left|\nabla \tilde{\sigma}_{\mathbf{i}}\right|^{2}\right) e^{-2 s \eta} d x d t\right. \\
& \left.\quad+\int_{Q_{\mathbf{t}}}\left(\left|\tilde{\sigma}_{\mathbf{t}}\right|^{2}+\left|\nabla \tilde{\sigma}_{\mathbf{t}}\right|^{2}\right) e^{-2 s \eta} d x d t+M_{t_{0}}^{2}\left(\tilde{v}_{\mathbf{m}}, \tilde{u}_{\mathbf{t}}, \tilde{\boldsymbol{w}}, \tilde{\boldsymbol{z}}\right)+M_{\omega}^{2}\left(\tilde{v}_{\mathbf{m}}, \tilde{u}_{\mathbf{h}}\right)+M_{\Gamma_{\text {ext }}}^{2}\left(\tilde{u}_{\mathbf{t}}\right)\right),
\end{aligned}
$$

for any $s>s_{0}$.

Proof. Applying Carleman estimate given by Theorem 2.3 to (3.32)-(3.33)-(3.34), we obtain

$$
\begin{aligned}
& \int_{Q_{\mathbf{h}}}\left(\left(\frac{s}{\ell(t)}\right)\left(\left|\partial_{t} \tilde{v}_{\mathbf{m}}^{(j)}\right|^{2}+\left|\operatorname{div}\left(\sigma_{\mathbf{m}} \nabla \tilde{v}_{\mathbf{m}}^{(j)}\right)\right|^{2}\right)+\left(\frac{s}{\ell(t)}\right)^{5}\left|\tilde{v}_{\mathbf{m}}^{(j)}\right|^{2}+\left(\frac{s}{\ell(t)}\right)^{3}\left|\nabla \tilde{v}_{\mathbf{m}}^{(j)}\right|^{2}\right) e^{-2 s \eta} d x d t \\
& +\int_{Q}\left(\left(\frac{s}{\ell(t)}\right)^{3}\left|\tilde{u}^{(j)}\right|^{2}+\left(\frac{s}{\ell(t)}\right)\left|\nabla \tilde{u}^{(j)}\right|^{2}\right) e^{-2 s \eta} d x d t \leqslant C\left(\int_{Q_{\mathbf{t}}}\left|\operatorname{div}\left(\tilde{\sigma}_{\mathbf{t}} \nabla \widehat{u}_{\mathbf{t}}^{(j)}\right)\right|^{2}\right) e^{-2 s \eta} d x d t \\
& +\int_{Q_{\mathbf{h}}}\left(\left(\frac{s}{\ell(t)}\right)^{2}\left(\left|\operatorname{div}\left(\tilde{\sigma}_{\mathbf{m}} \nabla \widehat{v}_{\mathbf{m}}^{(j)}\right)\right|^{2}+\left|\partial_{t}^{j} R(x, t)\right|^{2}\right)+\left(\left|\operatorname{div}\left(\tilde{\sigma}_{\mathbf{i}} \nabla \widehat{v}_{\mathbf{m}}^{(j)}\right)\right|^{2}+\left|\operatorname{div}\left(\tilde{\sigma}_{\mathbf{h}} \nabla \widehat{u}_{\mathbf{h}}^{(j)}\right)\right|^{2}\right)\right) e^{-2 s \eta} d x d t \\
& \left.+\int_{\Sigma_{\text {ext }}}\left(\frac{s}{\ell(t)}\right)\left|\nabla_{\tau} \tilde{u}_{\mathbf{t}}^{(j)}\right|^{2} e^{-2 s \eta} d x d t+\int_{Q_{\omega}}\left(\left(\frac{s}{\ell(t)}\right)^{5}\left|\tilde{v}^{(j)}\right|^{2}+\left(\frac{s}{\ell(t)}\right)^{3}\left|\tilde{u}_{\mathbf{h}}^{(j)}\right|^{2}\right) e^{-2 s \eta} d x d t\right) .
\end{aligned}
$$

From the condition (3.36), we get

$$
\begin{gathered}
\sum_{j=0}^{2}\left(\int_{Q_{\mathbf{h}}}\left(\left(\frac{s}{\ell(t)}\right)\left(\left|\partial_{t} \tilde{v}_{\mathbf{m}}^{(j)}\right|^{2}+\left|\operatorname{div}\left(\sigma_{\mathbf{m}} \nabla \tilde{v}_{\mathbf{m}}^{(j)}\right)\right|^{2}\right)+\left(\frac{s}{\ell(t)}\right)^{5}\left|\tilde{v}_{\mathbf{m}}^{(j)}\right|^{2}+\left(\frac{s}{\ell(t)}\right)^{3}\left|\nabla \tilde{v}_{\mathbf{m}}^{(j)}\right|^{2}\right) e^{-2 s \eta} d x d t\right. \\
\left.\quad+\int_{Q}\left(\left(\frac{s}{\ell(t)}\right)^{3}\left|\tilde{u}^{(j)}\right|^{2}+\left(\frac{s}{\ell(t)}\right)\left|\nabla \tilde{u}^{(j)}\right|^{2}\right) e^{-2 s \eta} d x d t\right) \\
\leqslant C\left(\int_{Q_{\mathbf{h}}}\left(\left(\frac{s}{\ell(t)}\right)^{2}\left(\left|\tilde{\sigma}_{\mathbf{m}}\right|^{2}+\left|\nabla \tilde{\sigma}_{\mathbf{m}}\right|^{2}\right)+\left(\left|\tilde{\sigma}_{\mathbf{i}}\right|^{2}+\left|\nabla \tilde{\sigma}_{\mathbf{i}}\right|^{2}\right)+\left(\left|\tilde{\sigma}_{\mathbf{h}}\right|^{2}+\left|\nabla \tilde{\sigma}_{\mathbf{h}}\right|^{2}\right)\right) e^{-2 s \eta} d x d t\right. \\
\left.+\int_{Q_{\mathbf{t}}}\left(\left|\tilde{\sigma}_{\mathbf{t}}\right|^{2}+\left|\nabla \tilde{\sigma}_{\mathbf{t}}\right|^{2}\right) e^{-2 s \eta} d x d t+\sum_{j=0}^{2} \int_{Q_{\mathbf{h}}}\left(\frac{s}{\ell(t)}\right)^{2}\left|\partial_{t}^{j} R(x, t)\right|^{2} e^{-2 s \eta} d x d t+M_{\omega}^{2}\left(\tilde{v}_{\mathbf{m}}, \tilde{u}_{\mathbf{h}}\right)+M_{\Gamma_{\mathrm{ext}}}^{2}\left(\tilde{u}_{\mathbf{t}}\right)\right) .
\end{gathered}
$$

We recall now that $\sigma_{\mathbf{m}}=\sigma_{\mathbf{i}} \sigma_{\mathbf{h}}^{-1} \sigma_{\mathbf{e}}$ where $\sigma_{\mathbf{h}}=\sigma_{\mathbf{i}}+\sigma_{\mathbf{e}}$ and by using (1.19), we obtain

$$
\tilde{\sigma}_{\mathbf{m}}=\left(\frac{\alpha}{1+\alpha}\right) \tilde{\sigma}_{\mathbf{i}} \quad \text { and } \quad \tilde{\sigma}_{\mathbf{h}}=(1+\alpha) \tilde{\sigma}_{\mathbf{i}}
$$

Thereafter, we get

$$
\begin{aligned}
& \sum_{j=0}^{2}\left(\int_{Q_{\mathbf{h}}}\left(\left(\frac{s}{\ell(t)}\right)\left(\left|\partial_{t} \tilde{v}_{\mathbf{m}}^{(j)}\right|^{2}+\left|\operatorname{div}\left(\sigma_{\mathbf{m}} \nabla \tilde{v}_{\mathbf{m}}^{(j)}\right)\right|^{2}\right)+\left(\frac{s}{\ell(t)}\right)^{5}\left|\tilde{v}_{\mathbf{m}}^{(j)}\right|^{2}+\left(\frac{s}{\ell(t)}\right)^{3}\left|\nabla \tilde{v}_{\mathbf{m}}^{(j)}\right|^{2}\right) e^{-2 s \eta} d x d t\right. \\
& \left.+\int_{Q}\left(\left(\frac{s}{\ell(t)}\right)^{3}\left|\tilde{u}^{(j)}\right|^{2}+\left(\frac{s}{\ell(t)}\right)\left|\nabla \tilde{u}^{(j)}\right|^{2}\right) e^{-2 s \eta} d x d t\right) \leqslant C\left(\int_{Q_{\mathbf{h}}}\left(\frac{s}{\ell(t)}\right)^{2}\left(\left|\tilde{\sigma}_{\mathbf{i}}\right|^{2}+\left|\nabla \tilde{\sigma}_{\mathbf{i}}\right|^{2}\right) e^{-2 s \eta} d x d t\right. \\
+ & \left.\int_{Q_{\mathbf{t}}}\left(\left|\tilde{\sigma}_{\mathbf{t}}\right|^{2}+\left|\nabla \tilde{\sigma}_{\mathbf{t}}\right|^{2}\right) e^{-2 s \eta} d x d t+\sum_{j=0}^{2} \int_{Q_{\mathbf{h}}}\left(\frac{s}{\ell(t)}\right)^{2}\left|\partial_{t}^{j} R(x, t)\right|^{2} e^{-2 s \eta} d x d t+M_{\omega}^{2}\left(\tilde{v}_{\mathbf{m}}, \tilde{u}_{\mathbf{h}}\right)+M_{\Gamma_{\mathrm{ext}}}^{2}\left(\tilde{u}_{\mathbf{t}}\right)\right) .
\end{aligned}
$$


Then, using Lemma 3.4, we have

$$
\begin{aligned}
\sum_{j=0}^{2} \int_{Q_{\mathbf{h}}}\left(\frac{s}{\ell(t)}\right)^{2}\left|\partial_{t}^{j} R(x, t)\right|^{2} e^{-2 s \eta} d x d t \leqslant & C\left(\sum_{j=0}^{2} \int_{Q_{\mathbf{h}}}\left(\frac{s}{\ell(t)}\right)^{2}\left|\partial_{t}^{j} \tilde{v}_{\mathbf{m}}(x, t)\right|^{2} e^{-2 s \eta} d x d t\right. \\
& \left.+\int_{Q_{\mathbf{h}}}\left(\frac{s}{\ell(t)}\right)^{2}\left(|\tilde{\mathbf{w}}(x, t)|^{2}+|\tilde{\mathbf{z}}(x, t)|^{2}\right) e^{-2 s \eta} d x d t\right) .
\end{aligned}
$$

In order to estimate $\tilde{\mathbf{w}}$ and $\tilde{\mathbf{z}}$, we apply Lemma 3.2. We obtain

$$
\begin{aligned}
& \int_{Q_{\mathbf{h}}} e^{-2 s \eta}\left(\frac{s}{\ell(t)}\right)^{2}\left(|\tilde{\mathbf{w}}(x, t)|^{2}+|\tilde{\mathbf{z}}(x, t)|^{2}\right) d x d t \\
& \quad \leqslant C\left(\int_{Q_{\mathbf{h}}} e^{-2 s \eta}\left(\frac{s}{\ell(t)}\right)^{2}\left(\left|\tilde{\mathbf{w}}\left(x, t_{0}\right)\right|^{2}+\left|\tilde{\mathbf{z}}\left(x, t_{0}\right)\right|^{2}\right) d x d t+D\right),
\end{aligned}
$$

where

with

$$
D:=\int_{Q_{\mathbf{h}}} s\left(\left|\partial_{t} \tilde{\mathbf{w}}(x, t)\right|^{2}+\left|\partial_{t} \tilde{\mathbf{z}}(x, t)\right|^{2}\right) e^{-2 s \eta} d x d t
$$

$$
\partial_{t} \tilde{\mathbf{w}}=\mathbf{F}\left(v_{\mathbf{m}}, \mathbf{w}\right)-\mathbf{F}\left(\widehat{v}_{\mathbf{m}}, \widehat{\mathbf{w}}\right)
$$

and

$$
\partial_{t} \tilde{\mathbf{z}}=\mathbf{G}\left(\bar{\varrho}, v_{\mathbf{m}}, \mathbf{w}, \mathbf{z}\right)-\mathbf{G}\left(\bar{\varrho}, \widehat{v}_{\mathbf{m}}, \widehat{\mathbf{w}}, \widehat{\mathbf{z}}\right) .
$$

Then, using the fact that $F$ and $G$ are locally Lipschitz, we get

$$
D \leqslant C\left(\int_{Q_{\mathbf{h}}} s\left|\tilde{v}_{\mathbf{m}}(x, t)\right|^{2} e^{-2 s \eta} d x d t+\int_{Q_{\mathbf{h}}} s\left(|\tilde{\mathbf{w}}(x, t)|^{2}+|\tilde{\mathbf{z}}(x, t)|^{2}\right) e^{-2 s \eta} d x d t\right) .
$$

Replacing now (3.43) in (3.42) and taking $s$ sufficiently large, we get

$$
\begin{aligned}
& \int_{Q_{\mathbf{h}}}\left(\frac{s}{\ell(t)}\right)^{2}\left(|\tilde{\mathbf{w}}(x, t)|^{2}+|\tilde{\mathbf{z}}(x, t)|^{2}\right) e^{-2 s \eta} d x d t \\
& \quad \leqslant C\left(\int_{Q_{\mathbf{h}}} s\left|\tilde{v}_{\mathbf{m}}(x, t)\right|^{2} e^{-2 s \eta} d x d t+\int_{Q_{\mathbf{h}}}\left(\frac{s}{\ell(t)}\right)^{2}\left(\left|\tilde{\mathbf{w}}\left(x, t_{0}\right)\right|^{2}+\left|\tilde{\mathbf{z}}\left(x, t_{0}\right)\right|^{2}\right) e^{-2 s \eta} d x d t\right) .
\end{aligned}
$$

Thereafter, from (3.41) and (3.44), we have

$$
\begin{aligned}
& \sum_{j=0}^{2} \int_{Q_{\mathbf{h}}}\left(\frac{s}{\ell(t)}\right)^{2}\left|\partial_{t}^{j} R(x, t)\right|^{2} e^{-2 s \eta} d x d t \\
& \quad \leqslant C\left(\sum_{j=0}^{2} \int_{Q_{\mathbf{h}}}\left(\frac{s}{\ell(t)}\right)^{2}\left|\tilde{v}_{\mathbf{m}}^{(j)}(x, t)\right|^{2} e^{-2 s \eta} d x d t+M_{t_{0}}^{2}\left(\tilde{v}_{\mathbf{m}}, \tilde{u}_{\mathbf{t}}, \tilde{\mathbf{w}}, \tilde{\mathbf{z}}\right)\right) .
\end{aligned}
$$

Subsequently, from (3.40) and (3.45) it is easily to deduce the desired estimate.

This completes the proof.

In the rest of the paper, we use the following notations

$$
\tilde{v}_{\mathbf{m}}^{(j)}\left(x, t_{0}\right)=\tilde{v}_{\mathbf{m}, 0}^{(j)}(x), \quad \tilde{u}_{\mathbf{h}}^{(j)}\left(x, t_{0}\right)=\tilde{u}_{\mathbf{h}, 0}^{(j)}(x), \quad \tilde{u}_{\mathbf{t}}^{(j)}\left(x, t_{0}\right)=\tilde{u}_{\mathbf{t}, 0}^{(j)}(x) .
$$

Lemma 3.6. There exist $s_{0}>0$ and $C>0$ such that the following estimate holds

$$
\begin{aligned}
& s \int_{\Omega_{\mathbf{h}}}\left(s^{3}\left|\tilde{v}_{\mathbf{m}, 0}^{(1)}\right|^{2}+s\left|\nabla \tilde{v}_{\mathbf{m}, 0}^{(1)}\right|^{2}\right) e^{-2 s \eta_{0}(x)} d x \leqslant C\left(\int_{Q_{\mathbf{h}}}\left(\frac{s}{\ell(t)}\right)^{2}\left(\left|\tilde{\sigma}_{\mathbf{i}}\right|^{2}+\left|\nabla \tilde{\sigma}_{\mathbf{i}}\right|^{2}\right) e^{-2 s \eta} d x d t\right. \\
& \left.\quad+\int_{Q_{\mathbf{t}}}\left(\left|\tilde{\sigma}_{\mathbf{t}}\right|^{2}+\left|\nabla \tilde{\sigma}_{\mathbf{t}}\right|^{2}\right) e^{-2 s \eta} d x d t+M_{\omega}^{2}\left(\tilde{v}_{\mathbf{m}}, \tilde{u}_{\mathbf{h}}\right)+M_{t_{0}}^{2}\left(\tilde{v}_{\mathbf{m}}, \tilde{u}_{\mathbf{t}}, \tilde{\boldsymbol{w}}, \tilde{\boldsymbol{z}}\right)+M_{\Gamma_{\text {ext }}}^{2}\left(\tilde{u}_{\mathbf{t}}\right)\right),
\end{aligned}
$$


ANALYSIS OF THE HEART-TORSO CONDUCTIVITY PARAMETERS RECOVERY INVERSE PROBLEM IN CARDIAC ELECTROPHYSIOLOGY ECG for any $s>s_{0}$.

Proof. Since $e^{-2 s \eta(x, 0)}=0$, we have

$$
\begin{aligned}
K_{1} & :=\int_{\Omega_{\mathbf{h}}}\left(\frac{s}{\ell\left(t_{0}\right)}\right)^{3}\left|\tilde{v}_{\mathbf{m}}^{(1)}\left(x, t_{0}\right)\right|^{2} e^{-2 s \eta_{0}(x)} d x=\int_{0}^{t_{0}} \int_{\Omega_{\mathbf{h}}} \frac{d}{d t}\left(\left(\frac{s}{\ell(t)}\right)^{3}\left|\tilde{v}_{\mathbf{m}}^{(1)}\right|^{2} e^{-2 s \eta(x, t)}\right) d x d t \\
& =\int_{0}^{t_{0}} \int_{\Omega_{\mathbf{h}}}\left(-2 s \partial_{t} \eta\left(\frac{s}{\ell(t)}\right)^{3}\left|\tilde{v}_{\mathbf{m}}^{(1)}\right|^{2}+3 s^{3}\left(\frac{\partial_{t} \ell(t)^{-1}}{\ell(t)^{2}}\right)\left|\tilde{v}_{\mathbf{m}}^{(1)}\right|^{2}+2 \tilde{v}^{(1)} \tilde{v}_{\mathbf{m}}^{(2)}\right) e^{-2 s \eta(x, t)} d x d t .
\end{aligned}
$$

Using the fact that $\left|\partial_{t} \ell(t)^{-1}\right| \leqslant C \ell(t)^{-2}$ and $\left|\partial_{t} \eta\right| \leqslant C_{\lambda} \ell^{-2}$, we deduce

$$
\left.\left|-2 s \partial_{t} \eta\left(\frac{s}{\ell(t)}\right)^{3}\right| \tilde{v}_{\mathbf{m}}^{(1)}\right|^{2}+3 s^{3}\left(\frac{\partial_{t} \ell(t)^{-1}}{\ell(t)^{2}}\right)\left|\tilde{v}_{\mathbf{m}}^{(1)}\right|^{2}+2 \tilde{v}_{\mathbf{m}}^{(1)} \tilde{v}_{\mathbf{m}}^{(2)} \mid \leqslant C s^{-1}\left(\frac{s}{\ell(t)}\right)^{5}\left(\left|\tilde{v}_{\mathbf{m}}^{(1)}\right|^{2}+\left|\tilde{v}_{\mathbf{m}}^{(2)}\right|^{2}\right) \text {. }
$$

By applying the Carleman estimate given in (3.37), we obtain

$$
\begin{aligned}
& \quad s \int_{\Omega_{\mathbf{h}}}\left(\frac{s}{\ell\left(t_{0}\right)}\right)^{3}\left|\tilde{v}_{\mathbf{m}}^{(1)}\left(x, t_{0}\right)\right|^{2} e^{-2 s \eta_{0}(x)} d x \leqslant C\left(\int_{Q_{\mathbf{h}}}\left(\frac{s}{\ell(t)}\right)^{2}\left(\left|\tilde{\sigma}_{\mathbf{i}}\right|^{2}+\left|\nabla \tilde{\sigma}_{\mathbf{i}}\right|^{2}\right) e^{-2 s \eta} d x d t\right. \\
& \left.\quad+\int_{Q_{\mathbf{t}}}\left(\left|\tilde{\sigma}_{\mathbf{t}}\right|^{2}+\left|\nabla \tilde{\sigma}_{\mathbf{t}}\right|^{2}\right) e^{-2 s \eta} d x d t+M_{t_{0}}^{2}\left(\tilde{v}_{\mathbf{m}}, \tilde{u}_{\mathbf{h}}, \tilde{\mathbf{w}}, \tilde{\mathbf{z}}\right)+M_{\omega}^{2}\left(\tilde{v}_{\mathbf{m}}, \tilde{u}_{\mathbf{h}}\right)+M_{\Gamma_{\mathrm{ext}}}^{2}\left(\tilde{u}_{\mathbf{t}}\right)\right) .
\end{aligned}
$$

In the other hand, we have

$$
\begin{aligned}
K_{2} & :=\int_{\Omega_{\mathbf{h}}}\left(\frac{s}{\ell\left(t_{0}\right)}\right)\left|\partial_{j} \tilde{v}_{\mathbf{m}}^{(1)}\left(x, t_{0}\right)\right|^{2} e^{-2 s \eta_{0}(x)} d x=\int_{0}^{t_{0}} \int_{\Omega_{\mathbf{h}}} \frac{d}{d t}\left(\left(\frac{s}{\ell(t)}\right)\left|\partial_{j} \tilde{v}_{\mathbf{m}}^{(1)}\right|^{2} e^{-2 s \eta(x, t)}\right) d x d t \\
& =\int_{0}^{t_{0}} \int_{\Omega_{\mathbf{h}}}\left(-2 s \partial_{t} \eta\left(\frac{s}{\ell(t)}\right)\left|\partial_{j} \tilde{v}_{\mathbf{m}}^{(1)}\right|^{2}+s \partial_{t} \ell(t)^{-1}\left|\partial_{j} \tilde{v}_{\mathbf{m}}^{(1)}\right|^{2}+2 \partial_{j} \tilde{v}_{\mathbf{m}}^{(1)} \partial_{j} \tilde{v}_{\mathbf{m}}^{(2)}\right) e^{-2 s \eta(x, t)} d x d t .
\end{aligned}
$$

Similar to (3.49), we find

$$
\left.\left|-2 s \partial_{t} \eta\left(\frac{s}{\ell(t)}\right)\right| \partial_{j} \tilde{v}_{\mathbf{m}}^{(1)}\right|^{2}+s \partial_{t} \ell(t)^{-1}\left|\partial_{j} \tilde{v}_{\mathbf{m}}^{(1)}\right|^{2}+2 \partial_{j} \tilde{v}_{\mathbf{m}}^{(1)} \partial_{j} \tilde{v}_{\mathbf{m}}^{(2)} \mid \leqslant C s^{-1}\left(\frac{s}{\ell(t)}\right)^{3}\left(\left|\nabla \tilde{v}_{\mathbf{m}}^{(1)}\right|^{2}+\left|\nabla \tilde{v}_{\mathbf{m}}^{(2)}\right|^{2}\right) .
$$

We apply again the Carleman estimate given in (3.37), we obtain

$$
\begin{aligned}
& s \int_{\Omega_{\mathbf{h}}}\left(\frac{s}{\ell\left(t_{0}\right)}\right)\left|\partial_{j} \tilde{v}_{\mathbf{m}}^{(1)}\left(x, t_{0}\right)\right|^{2} e^{-2 s \eta_{0}(x)} d x \leqslant C\left(\int_{Q_{\mathbf{h}}}\left(\frac{s}{\ell(t)}\right)^{2}\left(\left|\tilde{\sigma}_{\mathbf{i}}\right|^{2}+\left|\nabla \tilde{\sigma}_{\mathbf{i}}\right|^{2}\right) e^{-2 s \eta} d x d t\right. \\
& \left.\quad+\int_{Q_{\mathbf{t}}}\left(\left|\tilde{\sigma}_{\mathbf{t}}\right|^{2}+\left|\nabla \tilde{\sigma}_{\mathbf{t}}\right|^{2}\right) e^{-2 s \eta} d x d t+M_{t_{0}}^{2}\left(\tilde{v}_{\mathbf{m}}, \tilde{u}_{\mathbf{t}}, \tilde{\mathbf{w}}, \tilde{\mathbf{z}}\right)+M_{\omega}^{2}\left(\tilde{v}_{\mathbf{m}}, \tilde{u}_{\mathbf{h}}\right)+M_{\Gamma_{\mathrm{ext}}}^{2}\left(\tilde{u}_{\mathbf{t}}\right)\right) .
\end{aligned}
$$

By adding inequalities (3.50) and (3.53), we can deduce (3.47).

\subsection{Proof of the stability estimates.}

3.3.1. Estimate for $\tilde{\sigma}_{\mathbf{i}}$ and $\tilde{\sigma}_{\mathbf{e}}$. In this subsection, we derive an estimate which involves a relation between the difference of the intra-cellular conductivities $\sigma_{\mathbf{i}}$ and $\widehat{\sigma}_{\mathbf{i}}$, the extra-cellular conductivities $\sigma_{\mathbf{e}}$ and $\widehat{\sigma}_{\mathbf{e}}$ and the measures $M_{t_{0}}^{2}\left(\tilde{v}_{\mathbf{m}}, \tilde{u}_{\mathbf{t}}, \tilde{\mathbf{w}}, \tilde{\mathbf{z}}\right), M_{\omega}^{2}\left(\tilde{v}_{\mathbf{m}}, \tilde{u}_{\mathbf{h}}\right)$ and $M_{\Gamma_{\mathrm{ext}}}^{2}\left(\tilde{u}_{\mathbf{t}}\right)$.

Lemma 3.7. There exist constants $s_{0}$ and $C>0$ such that the following estimate holds

$$
\begin{aligned}
& \int_{\Omega_{\mathbf{h}}}\left(s^{4}\left(\left|\tilde{\sigma}_{\mathbf{i}}\right|^{2}+\left|\tilde{\sigma}_{\mathbf{e}}\right|^{2}\right)+s^{2}\left(\left|\nabla \tilde{\sigma}_{\mathbf{i}}\right|^{2}+\left|\nabla \tilde{\sigma}_{\mathbf{e}}\right|^{2}\right)\right) e^{-2 s \eta_{0}(x)} d x \leqslant C\left(\int_{\omega}\left(s^{4}\left|\tilde{\sigma}_{\mathbf{i}}\right|^{2}+s^{2}\left|\nabla \tilde{\sigma}_{\mathbf{i}}\right|^{2}\right) e^{-2 s \eta\left(x, t_{0}\right)} d x\right. \\
& \left.+\int_{\Omega_{\mathbf{t}}} s^{-1 / 2}\left(\left|\tilde{\sigma}_{\mathbf{t}}\right|^{2}+\left|\nabla \tilde{\sigma}_{\mathbf{t}}\right|^{2}\right) e^{-2 s \eta_{0}(x)} d x+M_{t_{0}}^{2}\left(\tilde{v}_{\mathbf{m}}, \tilde{u}_{\mathbf{t}}, \tilde{\boldsymbol{w}}, \tilde{\boldsymbol{z}}\right)+M_{\omega}^{2}\left(\tilde{v}_{\mathbf{m}}, \tilde{u}_{\mathbf{h}}\right)+M_{\Gamma_{e x t}}^{2}\left(\tilde{u}_{\mathbf{t}}\right)\right),
\end{aligned}
$$

for any $s \geqslant s_{0}$. 
Proof. By the first equation in (3.16) at a fixed time $t_{0}$, we get

$$
\operatorname{div}\left(\tilde{\sigma}_{\mathbf{m}} \nabla \hat{v}_{\mathbf{m}}\left(x, t_{0}\right)\right)=\tilde{v}_{\mathbf{m}}^{(1)}\left(x, t_{0}\right)-\operatorname{div}\left(\sigma_{\mathbf{m}} \nabla \tilde{v}_{\mathbf{m}}\left(x, t_{0}\right)\right)-R\left(x, t_{0}\right) .
$$

Then, we multiply (3.55) by the weight function $e^{-2 s \eta_{0}(x)}$, we integrate over $\Omega_{\mathbf{h}}$ and using (3.18), we obtain

$$
\begin{aligned}
\int_{\Omega_{\mathbf{h}}} s^{3}\left|\operatorname{div}\left(\tilde{\sigma}_{\mathbf{m}} \nabla \hat{v}_{\mathbf{m}}\left(x, t_{0}\right)\right)\right|^{2} e^{-2 s \eta_{0}(x)} d x \\
\quad \leqslant \int_{\Omega_{\mathbf{h}}} s^{3}\left(\left|\tilde{v}_{\mathbf{m}}^{(1)}\left(x, t_{0}\right)\right|^{2}+\mid \operatorname{div}\left(\left.\sigma_{\mathbf{m}} \nabla \tilde{v}_{\mathbf{m}}\left(x, t_{0}\right)\right|^{2}+\left|R\left(x, t_{0}\right)\right|^{2}\right) e^{-2 s \eta_{0}(x)} d x\right. \\
\quad \leqslant \int_{\Omega_{\mathbf{h}}} s^{3}\left|\tilde{v}_{\mathbf{m}}^{(1)}\left(x, t_{0}\right)\right|^{2} e^{-2 s \eta_{0}(x)} d x+M_{t_{0}}^{2}\left(\tilde{v}_{\mathbf{m}}, \tilde{u}_{\mathbf{t}}, \tilde{\mathbf{w}}, \tilde{\mathbf{z}}\right) .
\end{aligned}
$$

Thereafter, from (3.47), we deduce

$$
\begin{aligned}
& \int_{\Omega_{\mathbf{h}}} s^{3}\left|\operatorname{div}\left(\tilde{\sigma}_{\mathbf{m}} \nabla \hat{v}_{\mathbf{m}}\left(x, t_{0}\right)\right)\right|^{2} e^{-2 s \eta_{0}(x)} d x \leqslant C\left(\int_{Q_{\mathbf{h}}}\left(\frac{s}{\ell(t)}\right)^{2}\left(\left|\tilde{\sigma}_{\mathbf{i}}\right|^{2}+\left|\nabla \tilde{\sigma}_{\mathbf{i}}\right|^{2}\right) e^{-2 s \eta} d x d t\right. \\
& \left.\quad+\int_{Q_{\mathbf{t}}}\left(\left|\tilde{\sigma}_{\mathbf{t}}\right|^{2}+\left|\nabla \tilde{\sigma}_{\mathbf{t}}\right|^{2}\right) e^{-2 s \eta} d x d t+M_{\omega}^{2}\left(\tilde{v}_{\mathbf{m}}, \tilde{u}_{\mathbf{h}}\right)+M_{\Gamma_{\mathrm{ext}}}^{2}\left(\tilde{u}_{\mathbf{t}}\right)+M_{t_{0}}^{2}\left(\tilde{v}_{\mathbf{m}}, \tilde{u}_{\mathbf{t}}, \tilde{\mathbf{w}}, \tilde{\mathbf{z}}\right)\right) .
\end{aligned}
$$

By the Carleman estimate for the first order partial differential equation given by Lemma 3.1 with $y=\tilde{\sigma}_{\mathrm{m}}$, we get

$$
\int_{\Omega_{\mathbf{h}}} s^{4}\left|\tilde{\sigma}_{\mathbf{m}}\right|^{2} e^{-2 s \eta_{0}(x)} d x \leqslant C \int_{\Omega_{\mathbf{h}}} s^{3}\left|\operatorname{div}\left(\tilde{\sigma}_{\mathbf{m}} \nabla \widehat{v}_{\mathbf{m}}\left(x, t_{0}\right)\right)\right|^{2} e^{-2 s \eta_{0}(x)} d x+s^{4} \int_{\omega}\left|\tilde{\sigma}_{\mathbf{m}}\right|^{2} e^{-2 s \eta_{0}(x)} d x,
$$

where we have used that $\left|\nabla \beta(x) \cdot \nabla \tilde{v}\left(x, t_{0}\right)\right| \geqslant c_{0}$ in $\left(\Omega_{\mathbf{h}} \backslash \omega_{0}\right)$ and $\tilde{\sigma}_{\mathbf{m}}=0$ on $S$. Thereafter, by using (3.39) we obtain

$$
\int_{\Omega_{\mathbf{h}}} s^{4}\left|\tilde{\sigma}_{\mathbf{i}}\right|^{2} e^{-2 s \eta_{0}(x)} d x \leqslant C \int_{\Omega_{\mathbf{h}}} s^{3}\left|\operatorname{div}\left(\tilde{\sigma}_{\mathbf{m}} \nabla \widehat{v}_{\mathbf{m}}\left(x, t_{0}\right)\right)\right|^{2} e^{-2 s \eta_{0}(x)} d x+s^{4} \int_{\tilde{\omega}}\left|\tilde{\sigma}_{\mathbf{i}}\right|^{2} e^{-2 s \eta_{0}(x)} d x .
$$

Then, we obtain from (3.57) and (3.58) the following estimate

$$
\begin{array}{r}
\int_{\Omega_{\mathbf{h}}} s^{4}\left|\tilde{\sigma}_{\mathbf{i}}\right|^{2} e^{-2 s \eta_{0}(x)} d x \leqslant C\left(\int_{Q_{\mathbf{h}}}\left(\frac{s}{\ell(t)}\right)^{2}\left(\left|\tilde{\sigma}_{\mathbf{i}}\right|^{2}+\left|\nabla \tilde{\sigma}_{\mathbf{i}}\right|^{2}\right) e^{-2 s \eta} d x d t+\int_{Q_{\mathbf{t}}}\left(\left|\tilde{\sigma}_{\mathbf{t}}\right|^{2}+\left|\nabla \tilde{\sigma}_{\mathbf{t}}\right|^{2}\right) e^{-2 s \eta} d x d t\right. \\
\left.+s^{4} \int_{\omega}\left|\tilde{\sigma}_{\mathbf{i}}\right|^{2} e^{-2 s \eta_{0}(x)} d x+M_{\omega}^{2}\left(\tilde{v}_{\mathbf{m}}, \tilde{u}_{\mathbf{h}}\right)+M_{\Gamma_{\mathrm{ext}}}^{2}\left(\tilde{u}_{\mathbf{t}}\right)+M_{t_{0}}^{2}\left(\tilde{v}_{\mathbf{m}}, \tilde{u}_{\mathbf{t}}, \tilde{\mathbf{w}}, \tilde{\mathbf{z}}\right)\right) .
\end{array}
$$

Furthermore, we have

$$
\operatorname{div}\left(\partial_{j} \tilde{\sigma}_{\mathbf{m}} \nabla \hat{v}_{\mathbf{m}}\left(x, t_{0}\right)\right)=\partial_{j}\left(\operatorname{div}\left(\tilde{\sigma}_{\mathbf{m}} \nabla \hat{v}_{\mathbf{m}}\left(x, t_{0}\right)\right)-\operatorname{div}\left(\tilde{\sigma}_{\mathbf{m}} \nabla \partial_{j} \widehat{v}_{\mathbf{m}}\left(x, t_{0}\right)\right)\right.
$$

From (3.55), we get

$$
\operatorname{div}\left(\partial_{j} \tilde{\sigma}_{\mathbf{m}} \nabla \hat{v}_{\mathbf{m}}\left(x, t_{0}\right)\right)=\partial_{j} \tilde{v}_{\mathbf{m}}^{(1)}\left(x, t_{0}\right)-\partial_{j}\left(\operatorname{div}\left(\sigma_{\mathbf{m}} \nabla \tilde{v}_{\mathbf{m}}\left(x, t_{0}\right)\right)-\partial_{j} R\left(x, t_{0}\right)-\operatorname{div}\left(\tilde{\sigma}_{\mathbf{m}} \nabla \partial_{j} \hat{v}_{\mathbf{m}}\left(x, t_{0}\right)\right) .\right.
$$

Using now the following estimation

$$
\left|\nabla R\left(x, t_{0}\right)\right|^{2} \leqslant\left(\mid \tilde{v}_{\mathbf{m}}\left(x,\left.t_{0}\right|^{2}+\mid \tilde{\mathbf{w}}\left(x,\left.t_{0}\right|^{2}+\mid \tilde{\mathbf{z}}\left(x,\left.t_{0}\right|^{2}+\mid \nabla \tilde{v}_{\mathbf{m}}\left(x,\left.t_{0}\right|^{2}+\mid \nabla \tilde{\mathbf{w}}\left(x,\left.t_{0}\right|^{2}+\mid \nabla \tilde{\mathbf{z}}\left(x,\left.t_{0}\right|^{2}\right),\right.\right.\right.\right.\right.\right.
$$

we get

$$
\begin{aligned}
\int_{\Omega_{\mathbf{h}}} s\left|\operatorname{div}\left(\partial_{j} \tilde{\sigma}_{\mathbf{m}} \nabla \hat{v}_{\mathbf{m}, 0}\right)\right|^{2} e^{-2 s \eta_{0}(x)} d x \leqslant C\left(\int_{\Omega_{\mathbf{h}}} s\left|\partial_{j} \tilde{v}_{\mathbf{m}}^{(1)}\left(x, t_{0}\right)\right|^{2} e^{-2 s \eta_{0}(x)} d x\right. \\
\left.+\int_{\Omega_{\mathbf{h}}} s\left(\left|\tilde{\sigma}_{\mathbf{m}}\right|^{2}+\left|\nabla \tilde{\sigma}_{\mathbf{m}}\right|^{2}\right) e^{-2 s \eta_{0}(x)} d x+M_{t_{0}}^{2}\left(\tilde{v}_{\mathbf{m}}, \tilde{u}_{\mathbf{t}}, \tilde{\mathbf{w}}, \tilde{\mathbf{z}}\right)\right) .
\end{aligned}
$$


ANALYSIS OF THE HEART-TORSO CONDUCTIVITY PARAMETERS RECOVERY INVERSE PROBLEM IN CARDIAC ELECTROPHYSIOLOGY ECG From (3.47), we deduce

$$
\begin{aligned}
\int_{\Omega_{\mathbf{h}}} s\left|\operatorname{div}\left(\partial_{j} \tilde{\sigma}_{\mathbf{m}} \nabla \hat{v}_{\mathbf{m}}\left(x, t_{0}\right)\right)\right|^{2} e^{-2 s \eta_{0}(x)} d x \leqslant C\left(\int_{Q_{\mathbf{h}}}\left(\frac{s}{\ell(t)}\right)^{2}\left(\left|\tilde{\sigma}_{\mathbf{i}}\right|^{2}+\left|\nabla \tilde{\sigma}_{\mathbf{i}}\right|^{2}\right) e^{-2 s \eta} d x d t\right. \\
\left.\quad+\int_{Q_{\mathbf{t}}}\left(\left|\tilde{\sigma}_{\mathbf{t}}\right|^{2}+\left|\nabla \tilde{\sigma}_{\mathbf{t}}\right|^{2}\right) e^{-2 s \eta} d x d t+M_{\omega}^{2}\left(\tilde{v}_{\mathbf{m}}, \tilde{u}_{\mathbf{h}}\right)+M_{\Gamma_{\mathrm{ext}}}^{2}\left(\tilde{u}_{\mathbf{t}}\right)+M_{t_{0}}^{2}\left(\tilde{v}_{\mathbf{m}}, \tilde{u}_{\mathbf{t}}, \tilde{\mathbf{w}}_{,}, \tilde{\mathbf{z}}\right)\right)
\end{aligned}
$$

Applying again the Carleman estimate for the first order partial differential equation given by Lemma 3.1 with $y=\partial_{j} \tilde{\sigma}_{\mathbf{m}}$, we get

$$
\int_{\Omega_{\mathbf{h}}} s^{2}\left|\nabla \tilde{\sigma}_{\mathbf{m}}\right|^{2} e^{-2 s \eta_{0}(x)} d x \leqslant \int_{\Omega_{\mathbf{h}}} s\left|\operatorname{div}\left(\partial_{j} \tilde{\sigma}_{\mathbf{m}} \nabla \widehat{v}_{\mathbf{m}}\left(x, t_{0}\right)\right)\right|^{2} e^{-2 s \eta_{0}(x)} d x+\int_{\omega} s^{2}\left|\nabla \tilde{\sigma}_{\mathbf{m}}\right|^{2} e^{-2 s \eta_{0}(x)} d x,
$$

since $\partial_{j} \tilde{\sigma}_{\mathbf{m}}=0$ on $S$. Thereafter, by using again (3.39) we obtain

$$
\begin{aligned}
\int_{\Omega_{\mathbf{h}}} s^{2}\left(\left|\tilde{\sigma}_{\mathbf{i}}\right|^{2}+\left|\nabla \tilde{\sigma}_{\mathbf{i}}\right|^{2}\right) e^{-2 s \eta_{0}(x)} d x \leqslant C\left(\int_{\Omega_{\mathbf{h}}} s\left|\operatorname{div}\left(\partial_{j} \tilde{\sigma}_{\mathbf{m}} \nabla \hat{v}_{\mathbf{m}}\left(x, t_{0}\right)\right)\right|^{2} e^{-2 s \eta_{0}(x)} d x\right. & \left.+\int_{\omega} s^{2}\left(\left|\tilde{\sigma}_{\mathbf{i}}\right|^{2}+\left|\nabla \tilde{\sigma}_{\mathbf{i}}\right|^{2}\right) e^{-2 s \eta_{0}(x)} d x\right)(3.6
\end{aligned}
$$

Then, from (3.63) and (3.64) we get the following inequality

$$
\begin{aligned}
\int_{\Omega_{\mathbf{h}}} s^{2}\left(\left|\tilde{\sigma}_{\mathbf{i}}\right|^{2}+\right. & \left.\left|\nabla \tilde{\sigma}_{\mathbf{i}}\right|^{2}\right) e^{-2 s \eta_{0}(x)} d x \leqslant C\left(\int_{Q_{\mathbf{h}}}\left(\frac{s}{\ell(t)}\right)^{2}\left(\left|\tilde{\sigma}_{\mathbf{i}}\right|^{2}+\left|\nabla \tilde{\sigma}_{\mathbf{i}}\right|^{2}\right) e^{-2 s \eta} d x d t\right. \\
& +\int_{Q_{\mathbf{t}}}\left(\left|\tilde{\sigma}_{\mathbf{t}}\right|^{2}+\left|\nabla \tilde{\sigma}_{\mathbf{t}}\right|^{2}\right) e^{-2 s \eta} d x d t+\int_{\omega} s^{2}\left(\left|\tilde{\sigma}_{\mathbf{i}}\right|^{2}+\left|\nabla \tilde{\sigma}_{\mathbf{i}}\right|^{2}\right) e^{-2 s \eta_{0}(x)} d x \\
& \left.\quad+M_{\omega}^{2}\left(\tilde{v}_{\mathbf{m}}, \tilde{u}_{\mathbf{h}}\right)+M_{\Gamma_{\mathrm{ext}}}^{2}\left(\tilde{u}_{\mathbf{t}}\right)+M_{t_{0}}^{2}\left(\tilde{v}_{\mathbf{m}}, \tilde{u}_{\mathbf{t}}, \tilde{\mathbf{w}}_{,}, \tilde{\mathbf{z}}\right)\right)
\end{aligned}
$$

By collecting (3.59) and (3.65) the following estimation holds

$$
\begin{array}{r}
\int_{\Omega_{\mathbf{h}}}\left(s^{4}\left|\tilde{\sigma}_{\mathbf{i}}\right|^{2}+s^{2}\left|\nabla \tilde{\sigma}_{\mathbf{i}}\right|^{2}\right) e^{-2 s \eta_{0}(x)} d x \leqslant C\left(\int_{Q_{\mathbf{h}}}\left(\frac{s}{\ell(t)}\right)^{2}\left(\left|\tilde{\sigma}_{\mathbf{i}}\right|^{2}+\left|\nabla \tilde{\sigma}_{\mathbf{i}}\right|^{2}\right) e^{-2 s \eta} d x d t\right. \\
+\int_{Q_{\mathbf{t}}}\left(\left|\tilde{\sigma}_{\mathbf{t}}\right|^{2}+\left|\nabla \tilde{\sigma}_{\mathbf{t}}\right|^{2}\right) e^{-2 s \eta} d x d t+\int_{\omega}\left(s^{4}\left|\tilde{\sigma}_{\mathbf{i}}\right|^{2}+s^{2}\left|\nabla \tilde{\sigma}_{\mathbf{i}}\right|^{2}\right) e^{-2 s \eta_{0}(x)} d x \\
\left.+M_{t_{0}}^{2}\left(\tilde{v}_{\mathbf{m}}, \tilde{u}_{\mathbf{t}} \tilde{\mathbf{w}}, \tilde{\mathbf{z}}\right)+M_{\omega}^{2}\left(\tilde{v}_{\mathbf{m}}, \tilde{u}_{\mathbf{h}}\right)+M_{\Gamma_{\mathrm{ext}}}^{2}\left(\tilde{u}_{\mathbf{t}}\right)\right)
\end{array}
$$

Thereafter, applying Lemma 3.3, we get

$$
s^{2} \int_{Q_{\mathbf{h}}} \ell(t)^{-2}\left(\left|\tilde{\sigma}_{\mathbf{i}}\right|^{2}+\left|\nabla \tilde{\sigma}_{\mathbf{i}}\right|^{2}\right) e^{-2 s \eta(x, t)} d x d t \leqslant C s^{3 / 2} \int_{\Omega_{\mathbf{h}}}\left(\left|\tilde{\sigma}_{\mathbf{i}}\right|^{2}+\left|\nabla \tilde{\sigma}_{\mathbf{i}}\right|^{2}\right) e^{-2 s \eta_{0}(x)} d x,
$$

and

$$
\int_{Q_{\mathbf{t}}}\left(\left|\tilde{\sigma}_{\mathbf{t}}\right|^{2}+\left|\nabla \tilde{\sigma}_{\mathbf{t}}\right|^{2}\right) e^{-2 s \eta(x, t)} d x d t \leqslant C s^{-1 / 2} \int_{\Omega_{\mathbf{t}}}\left(\left|\tilde{\sigma}_{\mathbf{t}}\right|^{2}+\left|\nabla \tilde{\sigma}_{\mathbf{t}}\right|^{2}\right) e^{-2 s \eta_{0}(x)} d x .
$$

Then, from (3.66), (3.67) and (3.68), we get

$$
\begin{aligned}
& \int_{\Omega_{\mathbf{h}}}\left(s^{4}\left|\tilde{\sigma}_{\mathbf{i}}\right|^{2}+s^{2}\left|\nabla \tilde{\sigma}_{\mathbf{i}}\right|^{2}\right) e^{-2 s \eta_{0}(x)} d x \leqslant C\left(\int_{\omega}\left(s^{4}\left|\tilde{\sigma}_{\mathbf{i}}\right|^{2}+s^{2}\left|\nabla \tilde{\sigma}_{\mathbf{i}}\right|^{2}\right) e^{-2 s \eta\left(x, t_{0}\right)} d x\right. \\
& \left.+\int_{\Omega_{\mathbf{t}}} s^{-1 / 2}\left(\left|\tilde{\sigma}_{\mathbf{t}}\right|^{2}+\left|\nabla \tilde{\sigma}_{\mathbf{t}}\right|^{2}\right) e^{-2 s \eta_{0}(x)} d x+M_{t_{0}}^{2}\left(\tilde{v}_{\mathbf{m}}, \tilde{u}_{\mathbf{t}}, \tilde{\mathbf{w}}, \tilde{\mathbf{z}}\right)+M_{\omega}^{2}\left(\tilde{v}_{\mathbf{m}}, \tilde{u}_{\mathbf{h}}\right)+M_{\Gamma_{\mathrm{ext}}}^{2}\left(\tilde{u}_{\mathbf{t}}\right)\right) .
\end{aligned}
$$

Furthermore, using the fact that $\tilde{\sigma}_{\mathbf{i}}=\alpha(x) \tilde{\sigma}_{\mathbf{e}}$ which is given by (1.19), we have

$$
\int_{\Omega_{\mathbf{h}}}\left(s^{4}\left|\tilde{\sigma}_{\mathbf{e}}\right|^{2}+s^{2}\left|\nabla \tilde{\sigma}_{\mathbf{e}}\right|^{2}\right) e^{-2 s \eta_{0}(x)} d x \leqslant C \int_{\Omega_{\mathbf{h}}}\left(s^{4}\left|\tilde{\sigma}_{\mathbf{i}}\right|^{2}+s^{2}\left|\nabla \tilde{\sigma}_{\mathbf{i}}\right|^{2}\right) e^{-2 s \eta_{0}(x)} d x .
$$


Finally, From (3.69) and (3.70), we can deduce (3.54). This completes the proof.

3.3.2. Estimate for $\tilde{\sigma}_{t}$. In this subsection, we derive an estimate which involves a relation between the difference of the torso conductivities $\sigma_{\mathbf{t}}$ and $\hat{\sigma}_{\mathbf{t}}$ and the measure $M_{t_{0}}^{2}\left(\tilde{v}_{\mathbf{m}}, \tilde{u}_{\mathbf{t}}, \tilde{\mathbf{w}}, \tilde{\mathbf{z}}\right)$.

Lemma 3.8. There exist constants $s_{0}, C=C_{\lambda}>0$ such that for any $s \geqslant s_{0}$ the following estimate holds

$$
\int_{\Omega_{\mathbf{t}}}\left(s^{4}\left|\tilde{\sigma}_{\mathbf{t}}\right|^{2}+s^{2}\left|\nabla \tilde{\sigma}_{\mathbf{t}}\right|^{2}\right) e^{-2 s \eta_{0}(x)} d x \leqslant C M_{t_{0}}^{2}\left(\tilde{v}_{\mathbf{m}}, \tilde{u}_{\mathbf{t}}, \tilde{\boldsymbol{w}}, \tilde{z}\right) .
$$

Proof. We will follow the same steps in Lemma 3.7. Thus, we consider the following equation appearing in the linearized system (3.16) at a fixed time $t_{0}$ :

$$
\operatorname{div}\left(\tilde{\sigma}_{\mathbf{t}} \nabla \widehat{u}_{\mathbf{t}}\left(x, t_{0}\right)\right)=-\operatorname{div}\left(\sigma_{\mathbf{t}} \nabla \tilde{u}_{\mathbf{t}}\left(x, t_{0}\right)\right) .
$$

Then, we multiply (3.72) by the weight function $e^{-2 s \eta_{0}(x)}$ and we integrate over $\Omega_{\mathbf{t}}$, we get

$$
s^{3} \int_{\Omega_{\mathbf{t}}}\left|\operatorname{div}\left(\tilde{\sigma}_{\mathbf{t}} \nabla \widehat{u}_{\mathbf{t}}\left(x, t_{0}\right)\right)\right|^{2} e^{-2 s \eta_{0}(x)} d x \leqslant C M_{t_{0}}^{2}\left(\tilde{v}_{\mathbf{m}}, \tilde{u}_{\mathbf{t}}, \tilde{\mathbf{w}}, \tilde{\mathbf{z}}\right) .
$$

Applying now the Carleman estimate for the first order partial differential equation given by lemma 3.1 with $y=\tilde{\sigma}_{\mathbf{t}}$, we obtain the following inequality

$$
\begin{aligned}
s^{4} \int_{\Omega_{\mathbf{t}}}\left|\tilde{\sigma}_{\mathbf{t}}\right|^{2} e^{-2 s \eta_{0}(x)} d x & \leqslant s^{3} \int_{\Omega_{\mathbf{t}}}\left|\operatorname{div}\left(\tilde{\sigma}_{\mathbf{t}} \nabla \tilde{u}_{\mathbf{t}}\left(x, t_{0}\right)\right)\right|^{2} e^{-2 s \eta_{0}(x)} d x \\
& \leqslant C M_{t_{0}}^{2}\left(\tilde{v}_{\mathbf{m}}, \tilde{u}_{\mathbf{t}}, \tilde{\mathbf{w}}, \tilde{\mathbf{z}}\right) .
\end{aligned}
$$

On the other hand, considering the derivative with respect to the space of the equation (3.72), we get

$$
\operatorname{div}\left(\partial_{j} \tilde{\sigma}_{\mathbf{t}} \nabla \widehat{u}_{\mathbf{t}}\left(x, t_{0}\right)\right)=-\left(\operatorname{div}\left(\tilde{\sigma}_{\mathbf{t}} \nabla \partial_{j} \widehat{u}_{\mathbf{t}}\left(x, t_{0}\right)\right)+\partial_{j} \operatorname{div}\left(\sigma_{\mathbf{t}} \nabla \tilde{u}_{\mathbf{t}}\left(x, t_{0}\right)\right)\right) .
$$

We multiply now (3.75) by the weight function $e^{-2 s \eta_{0}(x)}$ and we integrate over $\Omega_{\mathbf{t}}$, we have

$$
\begin{aligned}
& s \int_{\Omega_{\mathbf{t}}}\left|\operatorname{div}\left(\partial_{j} \tilde{\sigma}_{\mathbf{t}} \nabla \widehat{u}_{\mathbf{t}}\left(x, t_{0}\right)\right)\right|^{2} e^{-2 s \eta_{0}(x)} d x \\
& \qquad C s \int_{\Omega_{\mathbf{t}}} \mid \operatorname{div}\left(\tilde{\sigma}_{\mathbf{t}} \nabla \partial_{j} \widehat{u}_{\mathbf{t}}\left(x, t_{0}\right)\right)+\partial_{j} \operatorname{div}\left(\left.\sigma_{\mathbf{t}} \nabla \tilde{u}_{\mathbf{t}}\left(x, t_{0}\right)\right|^{2} e^{-2 s \eta_{0}(x)} d x .\right.
\end{aligned}
$$

Furthermore, using the notations given by (3.35) and taking into account the condition (3.36), we get

$$
s \int_{\Omega_{\mathbf{t}}}\left|\operatorname{div}\left(\partial_{j} \tilde{\sigma}_{\mathbf{t}} \nabla \widehat{u}_{\mathbf{t}}\left(x, t_{0}\right)\right)\right|^{2} e^{-2 s \eta_{0}(x)} d x \leqslant C\left(\int_{\Omega_{\mathbf{t}}} s\left(\left|\tilde{\sigma}_{\mathbf{t}}\right|^{2}+\left|\nabla \tilde{\sigma}_{\mathbf{t}}\right|^{2}\right) e^{-2 s \eta_{0}(x)} d x+M_{t_{0}}^{2}\left(\tilde{v}_{\mathbf{m}}, \tilde{u}_{\mathbf{t}}, \tilde{\mathbf{w}}, \tilde{\mathbf{z}}\right)\right) .
$$

Moreover, we apply again the Carleman estimate for the first order partial differential equation given by lemma 3.1 with $y=\partial_{j} \tilde{\sigma}_{\mathbf{t}}$, we obtain the following estimate

$$
s^{2} \int_{\Omega_{\mathbf{t}}}\left|\nabla \tilde{\sigma}_{\mathbf{t}}\right|^{2} e^{-2 s \eta_{0}(x)} d x \leqslant C\left(\int_{\Omega_{\mathbf{t}}} s\left(\left|\tilde{\sigma}_{\mathbf{t}}\right|^{2}+\left|\nabla \tilde{\sigma}_{\mathbf{t}}\right|^{2}\right) e^{-2 s \eta_{0}(x)} d x+M_{t_{0}}^{2}\left(\tilde{v}_{\mathbf{m}}, \tilde{u}_{\mathbf{t}}, \tilde{\mathbf{w}}, \tilde{\mathbf{z}}\right)\right)
$$

Finally, summing (3.74) and (3.78) and for $s$ large, we get (3.71). This completes the proof. 
3.3.3. End of the proof of Theorem 1.2. In order to complete the proof of the stability result given by Theorem 1.2, we deduce from Lemma 3.7 and Lemma 3.8 the following estimate :

$$
\begin{gathered}
\int_{\Omega_{\mathbf{h}}}\left(s^{4}\left(\left|\tilde{\sigma}_{\mathbf{i}}\right|^{2}+\left|\tilde{\sigma}_{\mathbf{e}}\right|^{2}\right)+s^{2}\left(\left|\nabla \tilde{\sigma}_{\mathbf{i}}\right|^{2}+\left|\nabla \tilde{\sigma}_{\mathbf{e}}\right|^{2}\right)\right) e^{-2 s \eta_{0}(x)} d x+\int_{\Omega_{\mathbf{t}}}\left(s^{4}\left|\tilde{\sigma}_{\mathbf{t}}\right|^{2}+s^{2}\left|\nabla \tilde{\sigma}_{\mathbf{t}}\right|^{2}\right) e^{-2 s \eta_{0}(x)} d x \\
\quad \leqslant C\left(\int_{\omega}\left(s^{4}\left|\tilde{\sigma}_{\mathbf{i}}\right|^{2}+s^{2}\left|\nabla \tilde{\sigma}_{\mathbf{i}}\right|^{2}\right) e^{-2 s \eta\left(x, t_{0}\right)} d x+M_{t_{0}}^{2}\left(\tilde{v}_{\mathbf{m}}, \tilde{u}_{\mathbf{t}}, \tilde{\mathbf{w}}, \tilde{\mathbf{z}}\right)+M_{\omega}^{2}\left(\tilde{v}_{\mathbf{m}}, \tilde{u}_{\mathbf{h}}\right)+M_{\Gamma_{\mathrm{ext}}}^{2}\left(\tilde{u}_{\mathbf{t}}\right)\right) .
\end{gathered}
$$

Thereafter, by setting the weight function in $\Omega_{\mathrm{h}}$ and in $\Omega_{\mathrm{t}}$, we obtain the following estimate

$$
\begin{aligned}
& s^{2} e^{-2 s\left(\max _{\Omega_{\mathbf{h}}} \eta_{0}\right)}\left(\left\|\tilde{\sigma}_{\mathbf{i}}\right\|_{H^{1}\left(\Omega_{\mathbf{h}}\right)}^{2}+\left\|\tilde{\sigma}_{\mathbf{e}}\right\|_{H^{1}\left(\Omega_{\mathbf{h}}\right)}^{2}\right)+s^{2} e^{-2 s\left(\max _{\Omega_{\mathbf{t}}} \eta_{0}\right)}\left\|\tilde{\sigma}_{\mathbf{t}}\right\|_{H^{1}\left(\Omega_{\mathbf{t}}\right)}^{2} \\
& \leqslant C\left(s^{4} e^{-2 s\left(\min _{\Omega_{\mathbf{h}}} \eta_{0}\right)}\left\|\tilde{\sigma}_{\mathbf{i}}\right\|_{H^{1}(\omega)}^{2}+M_{t_{0}}^{2}\left(\tilde{v}_{\mathbf{m}}, \tilde{u}_{\mathbf{t}}, \tilde{\mathbf{w}}, \tilde{\mathbf{z}}\right)+M_{\omega}^{2}\left(\tilde{v}_{\mathbf{m}}, \tilde{u}_{\mathbf{h}}\right)+M_{\Gamma_{\mathrm{ext}}}^{2}\left(\tilde{u}_{\mathbf{t}}\right)\right) .
\end{aligned}
$$

Finally, we fix $s$ large enough and thus the proof of Theorem 1.2 is completed.

\section{ACKNOWLEDGEMENTS}

This work has been supported by EPICARD cooperative research program, funded by INRIA international laboratory LIRIMA. The LAMSIN researcher's work is supported on a regular basis by the Tunisian Ministry of Higher Education, Scientific Research and Technology. This work was also supported by the Agence Nationale de la Recherche (Grant IHU LIRYC ANR-10-IAHU-04).

\section{REFERENCES}

[1] Y. Abidi, M. Bellassoued, M. Mahjoub, and N. Zemzemi. On the identification of multiple space dependent ionic parameters in cardiac electrophysiology modelling. Inverse Problems, 34(3):035005, 2018.

[2] Y. Abidi, M. Bellassoued, M. Mahjoub and N. Zemzemi. Ionic parameters identification of an inverse problem of strongly coupled pdes system in cardiac electrophysiology using Carleman estimates. Mathematical Modelling of Natural Phenomena, 14(2):202, 2019.

[3] M. Bendahmane B. Ainseba and He. Yuan. Stability of conductivities in an inverse problem in the reaction-diffusion system in electrocardiology. Am. Inst. Math. Sci., 10 (2015) 369-385.

[4] L.Baudouin, E. Cerpa, E. Crépeau and A. Mercado. Lipschitz stability in an inverse problem for the kuramoto-sivashinsky equation. Applicable Analysis, 92(10):2084-2102, 2013.

[5] M. Bellassoued. Global logarithmic stability in inverse hyperbolic problem by arbitrary boundary observation. Inverse Problems, 20(4):1033, 2004.

[6] M. Bellassoued. Uniqueness and stability in determining the speed of propagation of second-order hyperbolic equation with variable coefficients. Applicable Analysis, 83(10):983-1014, 2004.

[7] M. Bellassoued and M. Yamamoto. Logarithmic stability in determination of a coefficient in an acoustic equation by arbitrary boundary observation. Journal de mathématiques pures et appliquées, 85(2):193-224, 2006.

[8] M. Bellassoued and M. Yamamoto. Determination of a coefficient in the wave equation with a single measurement. Applicable Analysis, 87(8):901-920, 2008.

[9] A. Benabdallah, M. Cristofol P.Gaitan and M. Yamamoto. Inverse problem for a parabolic system with two components by measurements of one component. Applicable Analysis, 88(5):683-709, 2009.

[10] A. Benabdallah, P. Gaitan, and J. Le Rousseau. Stability of discontinuous diffusion coefficients and initial conditions in an inverse problem for the heat equation. SIAM journal on control and optimization, 46(5):1849-1881, 2007.

[11] E. Beretta, C. Cavaterra, M.C. Cerutti, A. Manzoni, and L. Ratti. An inverse problem for a semilinear parabolic equation arising from cardiac electrophysiology. Inverse Problems, 33(10), 2017.

[12] A.L. Bugheim and M.V. Klibanov. Global uniqueness of class of multidimensional inverse problems. In Soviet Math. Dokl, volume 24, pages 244-247, 1981.

[13] A.L. Bukhgeim, J. Cheng, V. Isakov, and M. Yamamoto. Uniqueness in determining damping coefficients in hyperbolic equations. In Analytic Extension Formulas and their Applications, pages 27-46. Springer, 2001.

[14] A.L. Bukhgeĭm. Introduction to the theory of inverse problems, volume 19. Walter de Gruyter, 2000.

[15] T. Carleman. Sur un problème d'unicité pour les systèmes d'équations aux dérivées partielles à deux variables indépendantes. Friedländer, 1939.

[16] J.C. Clements, J. Nenonen, P. Li, and B. M. Horáček. Activation dynamics in anisotropic cardiac tissue via decoupling. Annals of biomedical engineering, 32(7):984-990, 2004. 
[17] L. Clerc. Directional differences of impulse spread in trabecular muscle from mammalian heart. The Journal of physiology, 255(2):335-346, 1976.

[18] P.C. Franzone and L.F. Pavarino. A parallel solver for reaction-diffusion systems in computational electrocardiology. Mathematical models and methods in applied sciences, 14(06):883-911, 2004.

[19] A. Doubova, A. Osses, and J-P. Puel. Exact controllability to trajectories for semilinear heat equations with discontinuous diffusion coefficients. ESAIM: Control, Optimisation and Calculus of Variations, 8:621-661, 2002.

[20] O.Yu. Emanuilov. Controllability of parabolic equations. Sbornik: Mathematics, 186(6):879, 1995.

[21] E. Fernández-Cara, M. González-Burgos, S. Guerrero, and J-P. Puel. Null controllability of the heat equation with boundary Fourier conditions: the linear case. ESAIM: Control, Optimisation and Calculus of Variations, 12(3):442-465, 2006.

[22] A.V Fursikov and O.Yu. Imanuvilov. Exact controllability of the Naiver-stokes and Bossiness equations. Russian Mathematical Surveys, 54(3):565, 1999.

[23] A.V. Fursikov and O.Yu Imanuvilov. Controllability of evolution equations. Number 34. Seoul National University, 1996.

[24] O.Yu Imanuvilov and M. Yamamoto. Lipschitz stability in inverse parabolic problems by the Carleman estimate. Inverse problems, 14(5):1229, 1998.

[25] J.P Keener and J. Sneyd. Mathematical physiology, volume 1. Springer, 1998.

[26] M.V. Klibanov. A class of inverse problems for nonlinear parabolic equations. Siberian Mathematical Journal, 27(5):698-708, 1986.

[27] M.V. Klibanov. On a class of inverse problems. In Doklady Akademii Nauk, volume 265, pages 1306-1309. Russian Academy of Sciences, 1982.

[28] M.V. Klibanov. Uniqueness in the large of solutions of inverse problems for a class of differential equations differ, 1985.

[29] J. Lassoued, M. Mahjoub, and N. Zemzemi. Stability results for the parameter identification inverse problem in cardiac electrophysiology. Inverse Problems, 32(11):115002, 2016.

[30] J.C. Neu and W. Krassowska. Homogenization of syncytial tissues. Critical reviews in biomedical engineering, 21(2):137199, 1993.

[31] D.M. Nguyen, P. Qian, T. Barry, and A. McEwan. The region-of-interest based measurement selection process for electrical impedance tomography in radiofrequency cardiac ablation with known anatomical information. Biomedical Signal Processing and Control, 56:101706, 2020.

[32] D.E. Roberts, L.T. Hersh, and A.M. Scher. Influence of cardiac fiber orientation on wavefront voltage, conduction velocity, and tissue resistivity in the dog. Circulation research, 44(5):701-712, 1979.

[33] D.E. Roberts and A.M. Scher. Effect of tissue anisotropy on extracellular potential fields in canine myocardium in situ. Circulation Research, 50(3):342-351, 1982.

[34] M.S Sulkin, J.I Laughner, S. Hilbert, S. Kapa, J. Kosiuk, P.Younan, I. Romero, A. Shuros, J.J Hamann, G. Hindricks, and A. Bollmann Novel measure of local impedance predicts catheter-tissue contact and lesion formation. Circulation: Arrhythmia and Electrophysiology, 11(4):e005831, 2018.

[35] J. Sundnes, G.T. Lines, X. Cai, B.F. Nielsen, K-A. Mardal, and A. Tveito. Computing the electrical activity in the heart, volume 1. Springer Science \& Business Media, 2007.

[36] K.T. Tusscher, R. Hren, and A.V. Panfilov. Organization of ventricular fibrillation in the human heart. Circulation research, 100(12):e87-e101, 2007.

[37] N.A. Trayanova. Whole-heart modeling: applications to cardiac electrophysiology and electromechanics. Circulation research, 108(1):113-128, 2011.

[38] L.Tung. A bi-domain model for describing ischemic myocardial dc potentials. $\mathrm{PhD}$ thesis, Massachusetts Institute of Technology, 1978.

[39] M. Veneroni. Reaction-diffusion systems for the microscopic cellular model of the cardiac electric field. Mathematical methods in the applied sciences, 29(14):1631-1661, 2006.

[40] M. Veneroni. Reaction-diffusion systems for the macroscopic bidomain model of the cardiac electric field. Nonlinear Analysis: Real World Applications, 10:849-868, 2009.

[41] B. Wu, L. Yan, Y. Gao, and Q. Chen. Carleman estimate for a linearized bidomain model in electrocardiology and its applications. Nonlinear Differential Equations and Applications NoDEA, 25(1):4, 2018.

[42] H. Yang and A. Veneziani. Estimation of cardiac conductivities in ventricular tissue by a variational approach. Inverse Problems, 31(11):115001, 2015.

E-mail address: abir.amridenit.utm.tn

Université de Tunis El Manar, Ecole Nationale D’Ingénieurs de Tunis, ENIT-LAMSiN, B.P. 37,1002 TuNIS, TUNISIA

E-mail address: mourad.bellassouedeenit.utm.tn 
ANALYSIS OF THE HEART-TORSO CONDUCTIVITY PARAMETERS RECOVERY INVERSE PROBLEM IN CARDIAC ELECTROPHYSIOLOGY ECG

Université de Tunis El Manar, Ecole Nationale D’Ingénieurs de Tunis, ENIT-Lamsin, B.P. 37, 1002 TUNIS, TUNISIA

E-mail address: moncef.mahjoubeenit.utm.tn

Université de Tunis El Manar, Ecole Nationale D’Ingénieurs de Tunis, ENIT-LAMSin, B.P. 37, 1002 TUNIS, TUNISIA

E-mail address: nejib.zemzemi@inria.fr

INRIA Bordeaux Sud-Ouest, Carmen project team. 200 Avenue de la vielle Tour 33405 Talence Cedex, FRANCE

IHU-LiRYC, AVEnue du Haut LVQue 33600 Pessac, France 